\title{
The Effect of Technology Assisted Teaching on Success in Mathematics and Geometry: A Meta-Analysis Study*
}

\author{
Hayati Çavuş ${ }^{*}$ \\ Computer Education and Instructional Technology, Faculty of Education, Van Yuzuncu Yil \\ University, Van, Turkey \\ ORCID: 0000-0001-5602-5221
}

\author{
Serap Deniz \\ Celaleddin Ökten Anatolan Imam Hatip High School, Gaziantep, Turkey \\ ORCID: 0000-0002-9458-1453
}

\begin{tabular}{|c|c|}
\hline Article history & ing the effectiveness of \\
\hline $\begin{array}{l}\text { Received: } \\
03.05 .2021\end{array}$ & $\begin{array}{l}\text { technology-assisted teaching to traditional method up on mathematical } \\
\text { and geometry success are combined through meta-analytical review }\end{array}$ \\
\hline $\begin{array}{l}\text { Received in revised form: } \\
12.07 .2021\end{array}$ & $\begin{array}{l}\text { method. For that purpose, articles, master's, and doctoral theses carried } \\
\text { out between the years } 2000-2016 \text { in Turkey are examined. } 98 \text { studies on } \\
\text { academic achievement meet the specified criteria and thus were included }\end{array}$ \\
\hline $\begin{array}{l}\text { Accepted: } \\
24.07 .2021\end{array}$ & $\begin{array}{l}\text { in the meta-analysis and the effect size between variables has been } \\
\text { demonstrated by assembling numerically the findings of these studies. }\end{array}$ \\
\hline Key words: & CMA 2.0, MS Office Excel 2010 programs were used in the analysis of \\
\hline $\begin{array}{l}\text { Academic } \\
\text { achievement; teaching } \\
\text { of } \\
\text { mathematics/geometry; } \\
\text { meta-analysis }\end{array}$ & $\begin{array}{l}\text { of the technology-assisted teaching is calculated as } 0,758 \text { on students' } \\
\text { mathematics achievement and as } 1,136 \text { on students' geometry } \\
\text { achievement. This study is based on the classification system according } \\
\text { to Cohen (1988). The effect sizes attained represent a medium effect size } \\
\text { for mathematics academic achievement and a very large size for } \\
\text { geometry achievement. Those both values are regarded as medium effect } \\
\text { size according to Cohen (1988). In addition, the comparative effect sizes } \\
\text { of the studies included in the study were calculated according to } \\
\text { techniques of implementation, level of education and learning fields. At } \\
\text { the end of the research, it is found that according to the traditional } \\
\text { learning method of technology-assisted mathematic and geometry } \\
\text { teaching is more effective in terms of achievement. It is concluded that } \\
\text { the effect size on the academic achievement of mathematic and } \\
\text { geometry does not differ according to time of implementation, level of } \\
\text { education, and learning fields. }\end{array}$ \\
\hline
\end{tabular}

\section{Introduction}

The studies on the ever-changing concepts of education and teaching frequently stem from searching for an answer to the question: "How can we provide a better education?" It will not be right to adopt status quo in the field of education and do nothing new in a world where

\footnotetext{
*Correspondency: hcavus@yyu.edu.tr
} 
needs change and increase day by day. It is not difficult to predict that the future teaching method will be oriented toward technological developments (Çavuş, 2006).

Today, dazzling advances in science, culture, and technology have affected many fields as well as the field of education. Many concepts from education programs to classroom structure such as teaching environments, educational tools, teacher, and student roles are rapidly changing and renewed with these technologies. Exciting developments in technology have provided new opportunities in mathematics teaching (Ertem Akbaş, 2019). This universal dimension offered by technology has significantly impacted on the question of "what should we teach and how?" Therefore, it is recommended to use technological facilities efficiently in designing learning environments where students can learn by doing (Güven, 2002). The needs to adapt education and teaching to the technological age on a constantly evolving and changing ground has been one of the top priorities in this context. In parallel with this, it was seen that the desired quality and contemporary goals could not be achieved with classical methods in teaching, and new searches were started.

In recent years, important mentality changes have occurred in mathematics education. As it is known, in traditional mathematics education, mathematical knowledge is presented to the student as small skills expected them to gain these skills with repeating and exercises. A student who must learn by memorizing many relations, rules, and symbols without comprehending, becomes unable to solve a problem (Olkun \& Toluk, 2001). However, as in every modern country, today's business world seeks people who have an analytical mindset, perceive problems correctly, and deliver creative solutions. For this reason, in mathematics education, raising individuals who know not only mathematics but also learn mathematics by doing mathematics has come to the fore. Therefore, the demonstration of the effectiveness of the Technology Assisted Instruction (TAI) method, which has become accepted among the teaching methods, has attracted the attention of the researchers, and led them to do many studies on this subject.

In the literature, although the number of studies on the effectiveness of TAI continues to increase over time, many independent studies came up with different findings from each other. In this case, it is considered important to combine the data obtained from these studies to give clear information to the target audience by gathering these independent studies under a single roof. Accordingly, the meta-analysis method, which allows us to make more general comments by combining these studies, emerges as a method that eliminates such problems.

Although there are many studies and applications on TAI in Turkey, any literature review allowing for large-scale generalizations in the area could not be found. It is thought that a metaanalysis study, which will be formed by the synthesis of the data obtained from the experimental studies that reveal the effect of technology-based learning in the fields of mathematics and geometry, conducted in Turkey, since 2000, will contribute to the literature by serving an important academic need in the field of technology-based learning and will shed light on the directions of future research. Combining the previous research results on the subject with the meta-analysis method and showing common results about its effects and practices in Turkey will contribute to reaching general conclusions. In this sense, this study will be useful in combining the research results to reveal whether the technological methods and tools used in mathematics and geometry lessons are effective in our country than the traditional teachercentered teaching method. Within the scope of the study, it is thought that the examination of meta-analytical effect size values and the effect of TAI on academic achievement in mathematics and geometry lessons can guide the plans and applications of technology supported education projects. In addition, the absence of a similar study showing the effect of 
technology-supported teaching methods on student achievement in mathematics and geometry increases the importance of this study. In this study, it was aimed to examine the effect of TAI on the students' achievement in mathematics and geometry lessons, considering the role of TAI in learning and teaching in mathematics and geometry within the scope of the literature. For this purpose, in this study, which examines how TAI affects the academic achievement of students compared to the traditional method, independent study results are combined with a meta-analysis method. Thus, it will be possible to see the big picture of the subject.

\section{Technology Supported Mathematics-Geometry Teaching and Academic Achievement}

Teaching such important branches of science is equally important. However, most students see mathematics and geometry as difficult science to learn and state that these are the subjects, they fear the most and do not like. Therefore, these courses are among the courses in which students are least successful. Carter and Good reported that the knowledge and skills usually developed in classes and determined by the grades, test scores, or both, appreciated by teachers, are called academic achievement (as cited in Arslan, 2008). To achieve success in teaching mathematics, students must have abilities such as reasoning, critical thinking, and problem-solving skills. Some international exams reveal that our country is insufficient in acquiring such skills with current mathematics education (Arslan, 2008). As a matter of fact, the academic achievements obtained in mathematics and geometry exams such as the International Mathematics and Science Trends Survey (TIMSS), the International Student Assessment Program (PISA), the International Reading Skills Development Project (PIRLS) are not very pleasant. This situation may be associated with the lack of attention of students in traditional settings, as the subject to be learned is not individualized (Ministry of Education, 2016). However, it is possible to attract attention to the lesson with individualized learning in a student-centered teaching environment prepared through software with information technology. In addition, it is possible to animate and model abstract content with computer technology. Thus, abstract concepts can be concretized and understood more easily by students. While National Council of Teachers of Mathematics (NCTM, 2004) shows one of the six items determined for quality mathematics education as the "technology principle", it supports and guides the use of technology. In the relevant literature, it is stated that technology should be used during the mathematics course, and this will help students learn mathematics (Ersoy, 2003; Ertem Akbaş, 2019; Ertem Akbaş, 2016; Nikolaou, 2000). In addition, although the use of technology in education is a requirement in general, mathematics specifically is a very suitable field for using technological facilities (Öksüz \& Ak, 2010). This situation is explained by the fact that computers used in mathematics education embody abstract concepts (MEB, 2016). For this reason, there is a general orientation and need for almost everyone to be mathematics and technology literate. Therefore, the efficient use of information technologies will make it possible for all students to reach mathematical thinking regardless of the level difference in the context of fulfilling certain mathematical skills of technology (Ersoy, 2003). In this respect, the tools used will lead students to use multimedia by saving them from time-consuming and repetitive calculations. Therefore, it should be important to teach students how to technologies effectively and appropriately use information and communication (MEB, 2016).

Even though information technologies and TAIs remind of computers, they are not limited to computers only. These technologies can be evaluated within the scope of hardware and software (Computer Algebra System (CAS)), Dynamic Geometry Software (DGY) in the fields of mathematics-geometry (Ersoy, 2003; Baki, 2002). Within the scope of this hardware and software, the importance of TAI in mathematics and geometry cannot be denied. In terms of facilitating the concretization and solution of the problems with tables, graphics, and symbols, TAIs help students. They help teachers and students to overcome some of the obstacles 
encountered during the problem-solving process. In addition, TAIs allow students to express numerical values with graphics, embody them with animations, use mathematical symbols, and examine the subject from different angles. For example, instead of discussing topics that are completely disconnected from their lives, such as finding or graphing the roots of a third-order polynomial function whose roots can be easily found, but rather it will allow students to discuss a more complex polynomial function that models a situation they may encounter in their daily lives which is meaningful to them. In this way, students will be able to think about the solution to the problem rather than finding ordinary roots and drawing graphics. Therefore, this thinking process will enable students to create meaningful information and deal with real problems, helping them understand that mathematics and geometry are an effort to find solutions to the human life problems (Durmuş, 2001). Similarly, it can be stated that the most important problem in teaching geometry is that the geometry course content is perceived as abstract and detached from daily life (Özkeleş-Çağlayan, 2010). However, it is complicated to explain subjects that require three-dimensional and spatial thinking skills with traditional methods. Parallel to this, since the geometric concepts are explained with two-dimensional tools such as books and pictures, the student faces some problems when moving from two dimensions to three dimensions. In this framework, the benefit of technology-supported applications is undeniable (Karal \& Berigel, 2008).

Only blackboard, chalk, or paper and pencil were used as tools for many years during mathematics-geometry education and training. Nowadays, information tools that will facilitate and assist the process have started to be used. Thanks to these informatics tools, TAIs bring along some developments such as creative thinking, problem solving skills, mathematical thinking instead of tiring minds empty and meaningless with memorization (Ersoy, 2003). As a matter of fact, students learn mathematics more profoundly and effectively and achieve academic success using information and communication technology (Ersoy, 2003). For this purpose, technological facilities should be utilized as much as possible to enrich students' mathematical understanding skills in mathematics classes (Hacısalihoğlu Karadeniz \& Akar, 2014; NCTM, 2004).

The content mentioned so far has dealt with the place and importance of TAI in mathematicsgeometry teaching and its effect on academic achievement. In this sense, the importance of presenting studies investigating the effect of technology support on academic achievement in mathematics and geometry teaching with meta-analysis (Y1ldı, 2009) method allows us to combine and provide more information by using one or more statistical methods, becomes evident.

\section{Purpose and Problem of the Research}

This study's main purpose is to synthesize the results obtained from experimental studies examining the effect of the TAI method on the academic achievement of students in mathematics and geometry lessons compared to the traditional method through meta-analysis. For this purpose, 98 studies were examined to evaluate how effective technology-supported teaching methods are in Turkey, and investigated the answers to the following questions within the scope of the research:

(1) What effect does TAI have on students' academic achievement in mathematics and geometry course areas?

(2) How do the effect sizes of the TAI techniques (animation, computer work, CD, computer software, smart board, calculator, simulation) differ? 
(3) When examined in terms of course areas (mathematics and geometry) in which the studies are conducted, what kind of difference is there between the effect sizes of the TAI method on academic achievement?

(4) How do the effect sizes of the TAI method differ in terms of students' education level (preschool, primary school, middle school, high school, university)?

\section{Method}

\section{Research Method}

This study is designed as a meta-analysis method, which is one of the quantitative research methods. Meta-analysis is a method that provides more reliable and clear results by presenting inferences based on standard numerical data instead of heuristic inferences used in other literature searches (Cohen et al., 2007). In addition, meta-analysis is the method used to combine and analyze the information called effect size quantitatively using data from individual studies (Camnalbur, 2008). In other words, meta-analysis is a method in which limited data can be examined in detail with a systematic review, and a single trend data can be obtained from scattered studies containing different characteristics (Binbay et al., 2011). The meta-Analysis, in short, is the analysis of analyses that combine the results of other studies consistently and harmoniously manner (Cohen et al., 2007). In this context, meta-analysis is the most suitable method for this study, which aims to combine and analyze in detail the studies conducted in our country between 2000-2016 to determine the effect of technology on student achievement in mathematics and geometry lessons. Besides, although the meta-analysis method has been a very popular in recent years, it is seen that the number of studies conducted with the meta-analysis method is quite low in Turkey, and the method has entered the domestic literature after the 2000s (Özcan, 2008). In this direction, it is thought that this study will contribute to the relevant literature.

\section{Data Collection (Application Process)}

Databases of ERIC (2015), Google Scholar (2015), and Turkish Academic Network and Information Center (ULAKBİM) (2015) scanned to access the articles published in journals, conference presentations, and papers obtained from the related subjects to answer the research questions, while Council of Higher Education in Turkey (CoHE) National Thesis Center was referred to for masters and doctoral dissertations. Apart from this, it has been tried to reach related studies by following the bibliography of similar studies that can be accessed from various search engines, libraries, and web pages of universities. The specified databases were first scanned in March 2015, and new studies were included in the research by re-scanning in January 2016.

Studies have been scanned using keywords such as "technology-supported teaching/learning / education", "technology-based/based teaching/learning/ education", "mathematics / geometry", "mathematics/geometry achievement", "mathematics achievement", "academic achievement", "technology-based learning / teaching / training", "technology-assisted learning / teaching / training", "effectiveness of technology-assisted education over mathematics achievement". 176 master's and doctoral theses and 107 articles in Turkey about the TAI between the years 2000 and 2016 have been reached. The studies found were included in the study pool according to the experimental studies' criteria in the scanned 16 years (2000-2016). Studies that do not have the necessary data for meta-analysis studies are eliminated. According to the selection features, a total of 98 studies including 11 doctoral theses, 70 master's theses and 17 articles were determined. It was seen that of these studies, 52 studies on mathematics and 46 studies on geometry research according to the academic achievement variable. While determining the course area to which the studies belong, the sub-learning area's acquisitions in which the 
application is carried out were taken as a basis. These studies included in the study are given in ANNEX-2.

\section{Inclusion Criteria}

Determining the criteria is the most critical point of meta-analysis. The criteria determine which studies will be included in the analysis and which will not. The criteria can be more than one. The most common criteria are experimental studies, data type, time frame, keywords, database, and publication type (Dinçer, 2014). The criteria for the inclusion of the research to be used for meta-analysis in this study are as follows:

- Aiming to determine the effectiveness level of technology-supported methods in teaching mathematics and geometry between 2000 and 2016.

- The subject sample is within the borders of Turkey.

- Studies that can be accessed from published theses, periodical academic journals, online academic journals, databases, academic studies presented in congresses and papers.

- Experimental studies with pretest-posttest control groups and the topics were taught to the experimental group by technology-supported methods and by traditional methods in the control group.

- Having the numerical data (arithmetic mean, standard deviation, sample numbers of the experimental group and the control group) is necessary for calculating the effect size.

\section{Data Coding}

Studies to be included in the meta-analysis were first saved in an electronic file format with a pdf extension, and a common data repository was created. These studies are grouped under three main headings in the Microsoft Excel worksheet: work identity, study content, and study data. Related data are presented under three headings as in Table 1.

Table 1. Coding Formats of Studies

\begin{tabular}{|c|c|c|}
\hline Study ID & Study Content & Statistics \\
\hline $\begin{array}{l}\text { Title } \\
\text { Authors } \\
\text { Year } \\
\text { City } \\
\text { City the Study Applied } \\
\text { Publication Type }\end{array}$ & $\begin{array}{l}\text { Learning Area (Mathematics } \\
\text { /Geometry) } \\
\text { Subject Applied } \\
\text { Learning Level } \\
\text { Method/Technique Used } \\
\text { Application Period } \\
\text { Dependent Variable (Achievement) }\end{array}$ & $\begin{array}{l}\text { Sample Size }(\mathrm{N}) \\
\text { Arithmetic Mean (X) } \\
\text { Standard Deviation (SD) }\end{array}$ \\
\hline
\end{tabular}

Data were obtained by opening a column for each subtitle and adding the investigated studies under the sections they belonged to in the coding form. To ensure coding reliability, the data were coded for a second time independently from the previous ones after a while. The coding form is given in ANNEX-1.

\section{Dependent Variables}

The research's dependent variable is the effect sizes of the TAI calculated based on the students' academic achievement scores in mathematics and geometry lessons.

\section{Study Characteristics}

Study characteristics are independent variables of meta-analysis. Study characteristics are coded to evaluate the relationships between the effect sizes, and they are used as explanatory variables in data analysis (Tarım, 2003). The operating characteristics added to the coding form (Annex-1) are listed as follows: 
- Publication Year

- Publication Type (master's thesis, doctoral dissertation, article)

- Learning Area (Mathematics /Geometry)

- Grade Level of the Sample

- Techniques used (software, computer, interactive board, projector, calculator, distance education... and others)

- Sample Size

Categorical Descriptive Statistics of the Studies Included in the Study

To calculate the effect size of the students' academic achievements in the mathematics and geometry lessons of TAI, 52 studies within the scope of mathematics lessons and 46 studies within the scope of geometry lessons which met the criteria determined were examined. The information about these studies, whose statistical significance level is accepted as $p=0.05$, are specified in the Table of Studies Included in the Meta-Analysis in Annex-2. Descriptive statistics of these studies for categorical independent variables are presented in Table 2.

Table 2. Frequency and Percentage Values for the Categorical Independent Variables of Studies that Contain Data on Academic Achievement

\begin{tabular}{|c|c|c|c|c|}
\hline \multirow[t]{2}{*}{ Variable } & \multicolumn{2}{|l|}{ Frequency } & \multicolumn{2}{|l|}{ Percentage } \\
\hline & Mathematics & Geometry & Mathematics & Geometry \\
\hline \multicolumn{5}{|l|}{ Year of Publication } \\
\hline 2002 & 1 & 0 & 1,92 & 0 \\
\hline 2003 & 0 & 1 & 0 & 2,17 \\
\hline 2004 & 1 & 0 & 1,92 & 0 \\
\hline 2005 & 2 & 0 & 3,85 & 0 \\
\hline 2006 & 2 & 2 & 3,85 & 4,34 \\
\hline 2007 & 6 & 5 & 11,53 & 10,87 \\
\hline 2008 & 8 & 4 & 15,38 & 8,69 \\
\hline 2009 & 7 & 4 & 13,46 & 8,69 \\
\hline 2010 & 5 & 7 & 9,61 & 15,21 \\
\hline 2011 & 5 & 7 & 9,61 & 15,21 \\
\hline 2012 & 5 & 7 & 9,61 & 15,21 \\
\hline 2013 & 3 & 5 & 5,76 & 10,87 \\
\hline 2014 & 6 & 3 & 11,53 & 6,52 \\
\hline 2015 & 0 & 1 & 0 & 2,17 \\
\hline \multicolumn{5}{|l|}{ Type of Publication } \\
\hline PhD Thesis & 9 & 2 & 17,30 & 4,34 \\
\hline Master's Thesis & 34 & 36 & 65,38 & 78,26 \\
\hline Paper & 9 & 8 & 17,30 & 17,39 \\
\hline \multicolumn{5}{|l|}{ Level of Application } \\
\hline Pre-school & 1 & 1 & 1,92 & 2,17 \\
\hline Elementary & 4 & 2 & 7,69 & 4,34 \\
\hline Middle School & 24 & 32 & 46,15 & 69,57 \\
\hline High School & 12 & 2 & 23,07 & 4,34 \\
\hline $\begin{array}{l}\text { University } \\
\text { (Undergraduate) }\end{array}$ & 11 & 3 & 21,15 & 6,52 \\
\hline \multicolumn{5}{|l|}{ Technology Used } \\
\hline Interactive board & 5 & 3 & 9,61 & 6,52 \\
\hline Computer & 15 & 7 & 25 & 13,04 \\
\hline Calculator & 2 & 0 & 3,85 & 0 \\
\hline Software applications & 20 & 36 & 42,30 & 80,43 \\
\hline Web Supported & 10 & 0 & 19,23 & 0 \\
\hline Total & 52 & 46 & & \\
\hline
\end{tabular}


When Table 2 is analysed according to the years in which the studies were conducted, it is observed that the number of studies containing data on the academic achievement variable among the studies included in the analysis increased after 2007. While studies investigating the effectiveness of TAI on the achievement of mathematics lessons focused especially between the years of 2007-2009, it is observed that the studies examining the achievement of the geometry lesson increased in 2009-2012. It is safe to say that the number of master's theses ranks first with a high rate of $71.43 \%$ in terms of the type of publication of the studies included in the analysis. Based on the grade level, the studies conducted at the secondary school $\left(5^{\text {th }}, 6^{\text {th }}\right.$, $7^{\text {th }}$, and $8^{\text {th }}$ grades) level have $57.17 \%$ of the studies included in the meta-analysis and constitute more than half of the studies included in the research. To examine the effectiveness of TAI methods on the academic achievement variable, it is seen that the most used technique in the implementation process of the studies, software programs with a rate of $42.30 \%$ for mathematics lessons and $80.43 \%$ for geometry lessons.

\section{Data Analysis}

The term that forms the nature of meta-analysis is the effect size. The effect size, which is also mentioned in the literature as the effect coefficient, is used in a study to give information about how the independent variable affects the dependent variable positively or negatively (Dinçer, 2014). For this purpose, the impact coefficients of the studies included in the research were calculated using the method developed by Hedges by using the Transaction Effectiveness Meta-Analysis in Group Comparison in the analysis of the data. The homogeneity test was performed for the effect size values found, and the "fixed effects" model was used in cases where homogeneity was achieved; otherwise, the "random effects" model was preferred. Since the significance level was taken as 0.05 in the studies included, 0.05 was determined as the significance level of the statistical analyses in this study. Below, various transformation formulas used in effect size calculations are given:

Table 3. Statistical Data Conversion Table

\begin{tabular}{|c|c|c|}
\hline $\begin{array}{l}\text { Statistics to } \\
\text { be } \\
\text { Converted }\end{array}$ & Formulas & Commentary \\
\hline $\begin{array}{l}\text { Means and } \\
\text { Standard } \\
\text { Deviations }\end{array}$ & $d=\frac{X_{e}-X_{c}}{S_{p}}$ & $\begin{array}{l}d=\text { Effect Size } \\
X_{e}=\text { Mean of Experimental Group } \\
X_{c}=\text { Mean of Control Group } \\
S_{p}=\text { Sum of Squares }\end{array}$ \\
\hline $\begin{array}{l}\text { Sum of } \\
\text { Squares }\end{array}$ & $S_{p}^{2}=\frac{\left(N_{e}-1\right) S^{2} e+\left(N_{c}-1\right) S^{2}{ }_{c}}{\left(N_{e}+N_{c}-2\right)}$ & $\begin{array}{l}N_{e}=\text { Size of Experimental Group } \\
N_{c}=\text { Size of Control Group } \\
S_{e}^{2}=\text { Variance of Experimental Group } \\
S_{c}^{2}=\text { Variance of Control Group } \\
S_{p}=\text { Sum of Squares }\end{array}$ \\
\hline $\mathrm{F}$ & $\begin{array}{c}d=\frac{2 t}{\sqrt{d f}} \\
d f=N_{e}+N_{c}-2\end{array}$ & $\begin{array}{l}\text { Used in independent or paired groups } \\
\text { t test. } \\
d=\text { Effect Size } \\
d f=\text { Degrees of freedom } \\
N_{e}=\text { Size of Experimental Group } \\
N_{c}=\text { Size of Control Group } \\
\text { Used only in the study } \\
\text { when given the F statistic value. } \\
d f=\text { Degrees of freedom } \\
d=\text { Effect Size }\end{array}$ \\
\hline & & $\begin{array}{l}\text { Used only in the study } \\
\text { when given the } r \text { statistic value. }\end{array}$ \\
\hline
\end{tabular}




\begin{tabular}{lll}
\hline$r$ & $d=\frac{2 r}{\sqrt{1-r^{2}}}$ & $\begin{array}{l}d=\text { Effect Size } \\
\operatorname{Var}(d)=d \text { Variance of Effect Size }\end{array}$ \\
Variance & $\operatorname{Var}(d)=\frac{N_{c}+N_{e}}{N_{c} \cdot N_{e}}+\frac{d^{2}}{2\left(N_{c}+N_{e}\right)}$ & $\begin{array}{l}\text { Serr }=d \text { Standard Error of Effect } \\
\text { Size }\end{array}$ \\
$\begin{array}{l}\text { Standard } \\
\text { Error }\end{array}$ & $\operatorname{Serr}=\sqrt{\operatorname{Var}(d)}$ & \\
\hline
\end{tabular}

The conversion formula used for effect size:

$d=$ Effect Size

$$
d=\frac{X_{e}-X_{c}}{S_{p}}
$$

$X_{e}=$ Mean of Experimental Group

$X_{c}=$ Mean of Control Group

$S_{p}=$ Sum of Squares

The conversion formula used for summed standard variance:

$$
S_{p}^{2}=\frac{\left(N_{e}-1\right) S_{e}^{2}+\left(N_{c}-1\right) S_{c}^{2}}{\left(N_{e}+N_{c}-2\right)}
$$

The conversion formula used for the summed standard deviation:

$N_{e}=$ Size of Experimental Group

$$
S_{p}=\sqrt{\frac{\left(N_{e}-1\right) S_{e}^{2}+\left(N_{c}-1\right) S_{c}^{2}}{\left(N_{e}+N_{c}-2\right)}}
$$

$N_{c}=$ Size of Control Group

$S_{e}^{2}=$ Variance of Experimental Group

$S_{c}^{2}=$ Variance of Control Group

$S_{p}=$ Sum of Squares

The conversion formula used in effect size calculations from $t$ test:

$$
\begin{gathered}
d=\frac{2 t}{\sqrt{d f}} \\
d f=N_{e}+N_{c}-2
\end{gathered}
$$

The conversion formula used in effect size calculations from $\mathrm{F}$ test:

$$
\begin{aligned}
& d=\frac{2 \sqrt{F}}{\sqrt{d f(\text { error })}} \\
& d f=d f(\text { error })
\end{aligned}
$$

While interpreting the calculated effect sizes, two effect size classification methods were used. One of them is one of the most frequently used classifications in the literature based on arithmetic averages developed by Cohen (1988). According to Cohen's, the other is the one developed by Thalheimer and Cook (2002), which is a relatively more detailed classification.

Classification of Cohen (1988) $d=0.20-0.50$ low level (small); $d=0.50-0.80$ medium level (medium); $d=0.80<\mathrm{d}$ is high level (large). In addition, the following comments are made for the effect sizes whose values vary between $-\infty$ and $\infty$ as a result of the calculations (Cohen, 1998): 
- If the effect size is zero, there is no difference between the experiment and control group.

- If the effect size is negative (-), the situation favors the control group, and the application has created an adverse effect.

- If the effect size is positive (+), it has a positive effect in favor of the experimental and application groups.

- The classification predicted by Thalheimer and Cook (2002) is $-0.15<\mathrm{d}<0.15$ negligible; $0.15<\mathrm{d}<0.40$ low level (small); $0.40<\mathrm{d}<0.75$ medium; $0.75<\mathrm{d}<1.10$ high (large); $1.10<\mathrm{d}<1.45$ very large; $1.45<\mathrm{d}$ is perfectly (huge).

To perform the calculations and graphics in this meta-analysis study, mainly the CMA (Comprehensive Meta-Analysis) statistical package program was used, but the MS Office Excel 2010 program was also used.

\section{Findings}

Within the scope of the study, quantitative studies comparing the effect of traditional methods with the technology-supported teaching (TAI) in mathematics and geometry education between 2000 and 2016 on academic achievement were examined. This examination led to the selection of 98 studies, including 11 doctoral theses, 70 master's thesis and 17 articles conducted in Turkey with relevant TAI methods. Afterwards, the effect sizes of these 98 (52 mathematics and 46 geometries) studies containing data on academic achievement variables suitable for inclusion criteria to do meta-analysis were analysed. In addition, data belonging to a sample group of 6202 people in total, 3546 of which are mathematics and 2656 of which are geometry, were examined within these studies. In this part of the study, the effect size values obtained by meta-analytical method and their interpretations are given.

\section{Findings of TAI's Effect on Academic Achievement Analysis in Mathematics and Geometry Lessons}

To find an answer to the first sub-problem of the study, "What kind of effect does TAI have on the academic achievement of students in mathematics and geometry?" The relevant data in the studies were analyzed via meta-analysis method. Effect size findings obtained from this analysis are presented under subheadings, viz. fixed and random effects model findings, forest plot, homogeneity test results, and publication bias findings.

\section{Uncombined Findings of the Effect Size Analysis of Studies According to Academic Achievement Variable}

Hedges D effect sizes of the studies containing data on the sub-problem of "What kind of effect does TAI have on the academic achievement of students in mathematics and geometry? "error values and the minimum and maximum values within $95 \%$ confidence interval are given in order, in Table 4 (mathematics lesson) and Table 5 (geometry lesson).

Table 4. Hedges D Effect Size Analysis Concerning Academic Achievement in Mathematics Course Uncombined Findings

\begin{tabular}{|c|c|c|c|c|c|}
\hline Study & $\begin{array}{l}\text { Effect Size } \\
\text { (d) }\end{array}$ & $\begin{array}{c}\text { Standart } \\
\text { Error }\end{array}$ & Variance & $\begin{array}{c}\text { Lower } \\
\text { Limit }\end{array}$ & $\begin{array}{l}\text { Upper } \\
\text { Limit }\end{array}$ \\
\hline Şen, 2010 & $-0,441$ & 0,331 & 0,109 & $-1,089$ & 0,207 \\
\hline Öner et all, 2014 & $-0,251$ & 0,336 & 0,113 & $-0,910$ & 0,408 \\
\hline Erginbaş, 2009 & $-0,179$ & 0,311 & 0,096 & $-0,788$ & 0,429 \\
\hline Çelik \& Çevik, 2011 & $-0,001$ & 0,259 & 0,067 & $-0,509$ & 0,507 \\
\hline Ünlü, 2007 & 0,013 & 0,232 & 0,054 & $-0,442$ & 0,467 \\
\hline
\end{tabular}




\begin{tabular}{|c|c|c|c|c|c|}
\hline Yigit, 2007 & 0,056 & 0,287 & 0,083 & $-0,507$ & 0,620 \\
\hline Esen, 2009 & 0,224 & 0,113 & 0,013 & 0,004 & 0,445 \\
\hline Tataroğlu, 2009 & 0,323 & 0,183 & 0,033 & $-0,036$ & 0,681 \\
\hline Aksoy, 2014 & 0,341 & 0,313 & 0,098 & $-0,272$ & 0,954 \\
\hline Aktümen, 2002 & 0,351 & 0,281 & 0,079 & $-0,199$ & 0,901 \\
\hline Şimşek, 2012 & 0,352 & 0,338 & 0,114 & - 0,309 & 1,014 \\
\hline Kabaca, 2006 & 0,366 & 0,358 & 0,128 & $-0,337$ & 1,068 \\
\hline Ăgaç, 2009 & 0,430 & 0,322 & 0,104 & $-0,201$ & 1,061 \\
\hline Karasel et all, 2009 & 0,441 & 0,265 & 0,070 & $-0,078$ & 0,960 \\
\hline Memişoğlu, 2005 & 0,497 & 0,195 & 0,038 & 0,115 & 0,879 \\
\hline Balkan, 2013 & 0,534 & 0,329 & 0,108 & $-0,110$ & 1,178 \\
\hline Aksoy et all, 2012 & 0,539 & 0,174 & 0,030 & 0,198 & 0,879 \\
\hline Turgut, 2010 & 0,558 & 0,219 & 0,048 & 0,128 & 0,988 \\
\hline Uygun, 2008 & 0,579 & 0,241 & 0,058 & 0,105 & 1,052 \\
\hline Özyurt ,2013 & 0,586 & 0,197 & 0,039 & 0,200 & 0,972 \\
\hline Aşıc1, 2014 & 0,587 & 0,256 & 0,066 & 0,085 & 1,090 \\
\hline Yorganc1, 2014 & 0,595 & 0,263 & 0,069 & 0,080 & 1,110 \\
\hline Arslan, 2008 & 0,600 & 0,261 & 0,068 & 0,089 & 1,111 \\
\hline Yorgancı \& Terzıoğlu, 2013 & 0,603 & 0,261 & 0,068 & 0,092 & 1,114 \\
\hline Önür, 2008 & 0,610 & 0,275 & 0,075 & 0,071 & 1,148 \\
\hline Gelibolu, 2008 & 0,628 & 0,263 & 0,069 & 0,112 & 1,145 \\
\hline Tuluk, 2007 & 0,668 & 0,366 & 0,134 & $-0,049$ & 1,385 \\
\hline Tural \& Sönmez, 2012 & 0,685 & 0,235 & 0,055 & 0,224 & 1,147 \\
\hline Aksoy, 2007 & 0,715 & 0,309 & 0,096 & 0,109 & 1,321 \\
\hline Kılıç, 2007 & 0,725 & 0,303 & 0,092 & 0,131 & 1,318 \\
\hline Çubuk, 2004 & 0,773 & 0,265 & 0,070 & 0,254 & 1,292 \\
\hline İnam, 2014 & 0,824 & 0,320 & 0,102 & 0,198 & 1,450 \\
\hline Özkök, 2010 & 0,884 & 0,284 & 0,081 & 0,327 & 1,440 \\
\hline Yazlık, 2011 & 0,884 & 0,179 & 0,032 & 0,532 & 1,236 \\
\hline Buran, 2005 & 0,961 & 0,211 & 0,045 & 0,547 & 1,375 \\
\hline Ekici, 2008 & 1,024 & 0,271 & 0,074 & 0,492 & 1,556 \\
\hline Şimşek, 2010 & 1,051 & 0,287 & 0,082 & 0,489 & 1,613 \\
\hline Gökcül, 2007 & 1,072 & 0,329 & 0,108 & 0,427 & 1,717 \\
\hline Doğan, 2009 & 1,105 & 0,254 & 0,065 & 0,606 & 1,603 \\
\hline Kutluca, 2009 & 1,111 & 0,383 & 0,147 & 0,360 & 1,862 \\
\hline Andıç, 2012 & 1,115 & 0,361 & 0,130 & 0,407 & 1,823 \\
\hline Özdoğan, 2008 & 1,120 & 0,238 & 0,057 & 0,652 & 1,587 \\
\hline Kepçeoğlu, 2010 & 1,226 & 0,339 & 0,115 & 0,562 & 1,890 \\
\hline Baytur, 2011 & 1,307 & 0,281 & 0,079 & 0,756 & 1,859 \\
\hline Bayturan \& Keşan, 2012 & 1,307 & 0,281 & 0,079 & 0,756 & 1,859 \\
\hline Alabay, 2006 & 1,497 & 0,336 & 0,113 & 0,837 & 2,156 \\
\hline İnce, 2008 & 1,503 & 0,278 & 0,077 & 0,957 & 2,048 \\
\hline Oğuz, 2008 & 1,568 & 0,267 & 0,071 & 1,044 & 2,092 \\
\hline Zengin, 2011 & 1,606 & 0,318 & 0,101 & 0,982 & 2,230 \\
\hline Kan, 2014 & 1,990 & 0,294 & 0,087 & 1,413 & 2,567 \\
\hline
\end{tabular}




\begin{tabular}{llllll}
\hline Firat, 2011 & 3,728 & 0,348 & 0,121 & 3,046 & 4,409 \\
\hline
\end{tabular}

When Table 4 is examined, it is observed that the effect sizes of 52 studies standardized according to the mathematics lesson's academic achievement variable vary between -0.441 and 3.728 .

Table 5. Hedges Effect Size Analysis Related to Academic Achievement in Geometry Course Uncombined Findings

\begin{tabular}{|c|c|c|c|c|c|}
\hline Study & $\begin{array}{l}\text { Effect Size } \\
\text { (d) }\end{array}$ & $\begin{array}{l}\text { Standart } \\
\text { Error }\end{array}$ & Variance & $\begin{array}{c}\text { Lower } \\
\text { Limit }\end{array}$ & $\begin{array}{l}\text { Upper } \\
\text { Limit }\end{array}$ \\
\hline Uzun, 2013 & 0,033 & 0,340 & 0,115 & $-0,633$ & 0,699 \\
\hline Akyar, 2010 & 0,050 & 0,253 & 0,064 & $-0,446$ & 0,545 \\
\hline Genç, 2010 & 0,478 & 0,240 & 0,058 & 0,008 & 0,948 \\
\hline Takunyacı, 2007 & 0,479 & 0,237 & 0,056 & 0,015 & 0,943 \\
\hline Uygan, 2011 & 0,525 & 0,289 & 0,083 & $-0,042$ & 1,091 \\
\hline Kurak, 2009 & 0,535 & 0,337 & 0,113 & $-0,124$ & 1,195 \\
\hline Özçakır, 2013 & 0,587 & 0,232 & 0,054 & 0,132 & 1,043 \\
\hline Şataf, 2010 & 0,587 & 0,296 & 0,088 & 0,007 & 1,168 \\
\hline Gülbağc1, 2009 & 0,607 & 0,303 & 0,092 & 0,012 & 1,202 \\
\hline Karakuş, 2008 & 0,648 & 0,287 & 0,082 & 0,085 & 1,211 \\
\hline Önal \& Demir, 2012 & 0,663 & 0,301 & 0,091 & 0,072 & 1,253 \\
\hline Demir, 2010 & 0,670 & 0,262 & 0,069 & 0,156 & 1,183 \\
\hline Akçayır, 2011 & 0,671 & 0,153 & 0,023 & 0,372 & 0,970 \\
\hline Öztürk, 2012 & 0,678 & 0,281 & 0,079 & 0,127 & 1,229 \\
\hline Egelioğlu, 2008 & 0,699 & 0,367 & 0,134 & $-0,020$ & 1,417 \\
\hline Özdemir \&Tabuk, 2003 & 0,754 & 0,242 & 0,058 & 0,280 & 1,227 \\
\hline Erdoğan, 2014 & 0,782 & 0,270 & 0,073 & 0,252 & 1,312 \\
\hline Özen, 2009 & 0,798 & 0,323 & 0,104 & 0,166 & 1,430 \\
\hline Sarı, 2012 & 0,800 & 0,295 & 0,087 & 0,221 & 1,379 \\
\hline Yıldiz, 2009 & 0,800 & 0,302 & 0,091 & 0,209 & 1,392 \\
\hline Efendioğlu, 2006 & 0,810 & 0,233 & 0,055 & 0,352 & 1,267 \\
\hline Baki \& Özpınar, 2007 & 0,830 & 0,250 & 0,063 & 0,340 & 1,320 \\
\hline Sarı, 2012 & 0,885 & 0,298 & 0,089 & 0,301 & 1,469 \\
\hline Birgi et all, 2007 & 0,961 & 0,317 & 0,100 & 0,340 & 1,582 \\
\hline Altın, 2012 & 0,991 & 0,329 & 0,108 & 0,346 & 1,636 \\
\hline Kaya et all, 2013 & 1,002 & 0,342 & 0,117 & 0,332 & 1,673 \\
\hline İçel, 2011 & 1,043 & 0,331 & 0,110 & 0,394 & 1,692 \\
\hline Uzun,2 014 & 1,088 & 0,327 & 0,107 & 0,448 & 1,728 \\
\hline Mercan, 2012 & 1,135 & 0,349 & 0,122 & 0,451 & 1,818 \\
\hline Öz, 2012 & 1,289 & 0,175 & 0,031 & 0,946 & 1,633 \\
\hline Karadeniz \&Akar, 2014 & 1,290 & 0,428 & 0,183 & 0,451 & 2,129 \\
\hline Toker, 2008 & 1,291 & 0,376 & 0,142 & 0,554 & 2,029 \\
\hline Tutak, 2008 & 1,298 & 0,352 & 0,124 & 0,607 & 1,989 \\
\hline Selçik \& Bilgici, 2011 & 1,352 & 0,384 & 0,148 & 0,599 & 2,106 \\
\hline Eryiğit, 2010 & 1,495 & 0,266 & 0,071 & 0,973 & 2,016 \\
\hline Küslü, 2015 & 1,510 & 0,305 & 0,093 & 0,912 & 2,108 \\
\hline
\end{tabular}




\begin{tabular}{lccccc}
\hline Topaloğlu, 2011 & 1,573 & 0,356 & 0,127 & 0,875 & 2,272 \\
Kaya, 2013 & 2,100 & 0,440 & 0,194 & 1,237 & 2,962 \\
Kaya, 2013 & 2,100 & 0,440 & 0,194 & 1,237 & 2,962 \\
Helvac1, 2010 & 2,192 & 0,309 & 0,096 & 1,586 & 2,798 \\
Aydoğa, 2007 & 2,228 & 0,219 & 0,048 & 1,799 & 2,658 \\
Tayan, 2011 & 2,355 & 0,342 & 0,117 & 1,685 & 3,026 \\
Abdüsselam,2006 & 2,589 & 0,593 & 0,351 & 1,428 & 3,751 \\
Budak, 2010 & 2,644 & 0,351 & 0,123 & 1,956 & 3,331 \\
Kesici, 2011 & 2,916 & 0,518 & 0,268 & 1,901 & 3,930 \\
Gündüz et all, 2007 & 3,124 & 0,263 & 0,069 & 2,609 & 3,639 \\
\hline
\end{tabular}

When Table 5 is examined, it is seen that the standardized effect sizes of 46 studies according to the academic achievement variable in the geometry lesson vary between 0.033 and 3.124 values. The frequency and percentage values of these studies' effect size aspects, in which TAI was examined in terms of academic achievement, are given in Table 6.

Table 6. Frequency and Percentage Table for Effect Size According to Academic Achievement Variable

\begin{tabular}{|c|c|c|c|c|}
\hline \multirow{2}{*}{ Effect Size Direction } & \multicolumn{2}{|l|}{ Frequency } & \multicolumn{2}{|l|}{ Percentage } \\
\hline & Mathematics & Geometry & Mathematics & Geometry \\
\hline 0 (Zero) & 0 & & 0 & 0 \\
\hline+ (Positive) & 48 & & 92.30 & 100 \\
\hline - (Negative) & 4 & & 7.69 & 0 \\
\hline
\end{tabular}

Looking at the effect sizes in Table 6, it is seen that 48 studies (92.3\%) have positive, and 4 studies $(7.69 \%)$ have negative effect sizes for mathematics. When it comes to the geometry lesson, it is seen that all 46 studies have positive effect sizes. The positive effect size values indicate that the academic achievement value in these studies favors the experimental group, depending on the degree of effect size. If the value of the effect size is negative, it reveals that the achievement scores in the studied study are in favor of the control group, depending on the effect size (Wolf, 1986). Based on this result, a negative effect size percentage being 7.69\% shows that the result favors the control group in these studies. In other words, these data show that student achievement, which is the studied variable, is in favor of TAI from the aspect of the effect size.

The effect sizes of the studies examined according to the academic achievement variable in the study were categorized based on Cohen's (1988) classification. Since the lower limit value in the effect size classification of Cohen (1988) is 0.20, it can be said that "no statistically significant effect has been found" for studies whose effect size is below this value. Effect sizes of 6 studies in mathematics (Şen, 2010; Öner et al., 2014; Erginbaş, 2009; Çelik \& Çevik, 2011; Ünlü, 2007; Yiğit 2007) and 2 studies in geometry (Uzun, 2013; Akyar, 2010) remained below this value. The frequency and percentage values of studies above 0.20 are listed in Table 7 below: 
Table 7. Frequency and Percentage Table of Cohen's Classification of Effect Size According to Academic Achievement Variable

\begin{tabular}{|c|c|c|c|c|c|c|}
\hline \multirow{2}{*}{$\begin{array}{l}\text { Effect } \\
\text { Level }\end{array}$} & \multirow[t]{2}{*}{ Size } & \multicolumn{2}{|l|}{ Frequency } & \multicolumn{3}{|c|}{ Percentage } \\
\hline & & Mathematics & Geometry & \multicolumn{2}{|c|}{ Mathematics } & Geometry \\
\hline Small & & 9 & & 17.30 & 4.35 & \\
\hline Medium & & 14 & & 32.69 & 30.43 & \\
\hline Large & & 20 & & 38.46 & 60.86 & \\
\hline
\end{tabular}

According to Thalheimer and Cook's (2002) more detailed scale, effect sizes of these studies were classified. In this classification, the effect sizes of 3 studies (Şen, 2010; Öner et al., 2014; Erginbaş, 2009) in the field of mathematics, and the effect sizes of 0 (zero) study in the geometry field was seen below the lower limit of Thalheimer's and Cook's scale, -0.15 . Since they are not within the confidence interval to which the effect size belongs, we can say that TAI does not significantly affect academic achievement in these studies. Effect size, frequency, and percentage values of the other studies are listed in Table 8 below:

Table 8. Frequency and Percentage Table of the More Detailed Classification of Effect Size According to Academic Achievement Variable (Thalheimer \& Cook, 2002)

\begin{tabular}{lllll}
\hline \multirow{2}{*}{ Effect Size } & Frequency & \multicolumn{3}{l}{ Percentage } \\
\cline { 2 - 5 } & Mathematics & Geometry & Mathematics & Geometry \\
\hline Negligible & 3 & 2 & 5.77 & 4.34 \\
Low & 6 & 0 & 11.54 & 0.00 \\
Medium & 19 & 13 & 36.54 & 28.26 \\
High & 9 & 13 & 11.54 & 28.26 \\
Very High & 6 & 6 & 11.54 & 13.04 \\
Excellent & 6 & 12 & 11.54 & 26.08 \\
\hline
\end{tabular}

In Table 8, based on Thalheimer and Cook's (2002) more detailed effect size classification, it was seen that the highest frequency for the effect size in the mathematics lesson is at a medium level with 19 studies (36.54\%), and was perfect in the geometry lesson, with 12 studies $(26.08 \%)$. In this context, it can be said that in most of the studies within this analysis, technology-assisted teaching has a greater effect on student achievement than traditional teaching.

\section{Forest Graph of Studies According to Academic Achievement Variable}

Figure 1 and Figure 2 show the forest graph of the studies included in the study according to the academic achievement variable in mathematics and geometry lessons, respectively. 


\begin{tabular}{|c|c|c|c|c|c|}
\hline \multirow[t]{2}{*}{ 8tudy name } & \multirow[b]{2}{*}{ Hedpes's } & \multicolumn{4}{|c|}{ Batistics for each study } \\
\hline & & $\begin{array}{l}\text { Bend ard } \\
\text { error }\end{array}$ & Verlan $\alpha$ & $\begin{array}{c}\text { Lower } \\
\text { Ilint }\end{array}$ & $\begin{array}{l}\text { Upper } \\
\text { ilinit }\end{array}$ \\
\hline AFEAN 208 & 0.500 & 02 & 0088 & 0,009 & 1.111 \\
\hline ECNNBEZ209 & .0179 & $0.3+1$ & 009 & .0788 & 000 \\
\hline TUUK 207 & 0008 & 035 & 0.134 & .0049 & 1395 \\
\hline BAYTR. I 11 & 1307 & 028 & 0079 & 0.75 & 1899 \\
\hline RTUCA 209 & 1.111 & 035 & 0.18 & 0380 & 138 \\
\hline KABACA200 & 037 & 038 & 0.12 & .035 & 1088 \\
\hline AVSOY 207 & 0.715 & 030 & $\cos 8$ & 0.109 & 1 푀 \\
\hline OZYRT:Z13 & 0506 & 0.13 & 0039 & 0200 & $05 \pi / 2$ \\
\hline YAZXX211 & 0.84 & 0.18 & $\cos$ & 052 & 126 \\
\hline SHIEXI12 & 035 & 0.35 & 0.114 & .0309 & 1014 \\
\hline AGAC 2009 & 0.430 & 03 & 0.104 & .021 & 1051 \\
\hline KEREQQU 210 & 125 & 0.35 & 0.115 & $0 \%$ & 1290 \\
\hline ZRVON 211 & 1.906 & 038 & 0.101 & 002 & 230 \\
\hline CEKCEK 211 & .0001 & 020 & 608 & .0509 & 0307 \\
\hline SONUIE. AFTाUT & 0.505 & 023 & $\cos$ & 0.24 & 1.17 \\
\hline KAFAE AVA TEER 209 & 0.44 & 025 & 000 & .0078 & 0000 \\
\hline AVSOYCAUKCNAR Z12 & 0539 & 0.174 & 0030 & 0.138 & 0879 \\
\hline $0064 \times 209$ & 1.105 & 028 & COE & 0,506 & 1900 \\
\hline BAMTUAN VESAN.T12 & 1307 & 028 & 0079 & 0.73 & 1939 \\
\hline YORGANC.TEROQU X13 & 0.003 & 02 & 0088 & 0.09 & 1.114 \\
\hline $03: 208$ & $1 \% 8$ & 0.28 & 0001 & 104 & 2092 \\
\hline VGT2OT & 0,05 & 0.8 & 0083 & .0557 & 080 \\
\hline MaIİau 205 & 0.487 & $0.1 \Xi$ & 008 & 0.115 & 0679 \\
\hline TATAFOQU 209 & 033 & 0.13 & 0033 & .0036 & 0681 \\
\hline Exav. 709 & $0=2$ & 0.18 & 0013 & 0.004 & 045 \\
\hline 0 T00G4N 208 & 1.12 & $0.2 \pi$ & 008 & $0=2$ & 1587 \\
\hline ALSAY 206 & 1.487 & 0.35 & 0.113 & 0.58 & 2136 \\
\hline BRAN ZI5 & 0901 & $0.2+1$ & 0045 & 059 & 135 \\
\hline $020 \times 210$ & 0884 & 0.34 & 0081 & 037 & 140 \\
\hline$B \times C .208$ & 100 & $02 \pi$ & 0074 & 0.482 & 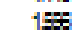 \\
\hline BAXSAN 213 & 0.534 & 039 & 0.108 & .0 .110 & 1.178 \\
\hline NuC 207 & 0.75 & 0.36 & $\cos 2$ & 0.131 & 1318 \\
\hline GEВบบ 208 & $0 \leqslant 8$ & 025 & 000 & 0.112 & 1.145 \\
\hline UNGIN 208 & $05 \sqrt{9}$ & 024 & 0058 & 0.105 & 105 \\
\hline TuRar.210 & $0=0$ & 028 & 0048 & 0.12 & 0988 \\
\hline TUA. BXNIEZ I12 & 0.605 & 0.25 & coss & 0.24 & 1.16 \\
\hline ARTOMBN 202 & 0351 & 029 & 0009 & .0199 & 0901 \\
\hline Goval 207 & $100 / 2$ & 039 & 0.108 & 0.47 & 1777 \\
\hline $\mathrm{NCE} 20 \mathrm{C}$ & 1.503 & $0 \pi$ & $00 \mathrm{OT}$ & 0.97 & 2048 \\
\hline $\operatorname{sen} 210$ & .044 & 033 & 0.109 & -1009 & 027 \\
\hline Busex 210 & 1.051 & 0.5 & 000 & 0.429 & 1813 \\
\hline FRAT.211 & $3 \pi 8$ & $0: 38$ & 0.21 & 3045 & 4.499 \\
\hline CUBX 2I4 & $0 \pi 73$ & 025 & 000 & 0284 & 120 \\
\hline $\mathrm{ANDC} 212$ & 1.115 & 098 & 0130 & 0.407 & 133 \\
\hline ovo 207 & 0.013 & 025 & 0054 & .040 & 048 \\
\hline ONR $2 \mathrm{Zz}$ & 0.510 & $0 . \overline{55}$ & 0075 & 0,071 & 1.148 \\
\hline No4. 214 & 084 & 037 & 010 & 0.138 & 1,1850 \\
\hline AVSY 214 & 0341 & 0.38 & 008 & $.0 \pi / 2$ & 0054 \\
\hline $\mathrm{ABC} .214$ & 057 & 025 & 000 & 0005 & 1090 \\
\hline KAN 214 & 190 & 02 & 000 & 1413 & 280 \\
\hline OER YDRM BAFE214 & .0251 & 035 & 0113 & .0910 & 0408 \\
\hline Yorganc 214 & $0: 395$ & 025 & 000 & 0000 & 1.110 \\
\hline & 0687 & 005 & 0001 & 0,518 & $07 x$ \\
\hline
\end{tabular}

Hedpes's g and 96\%

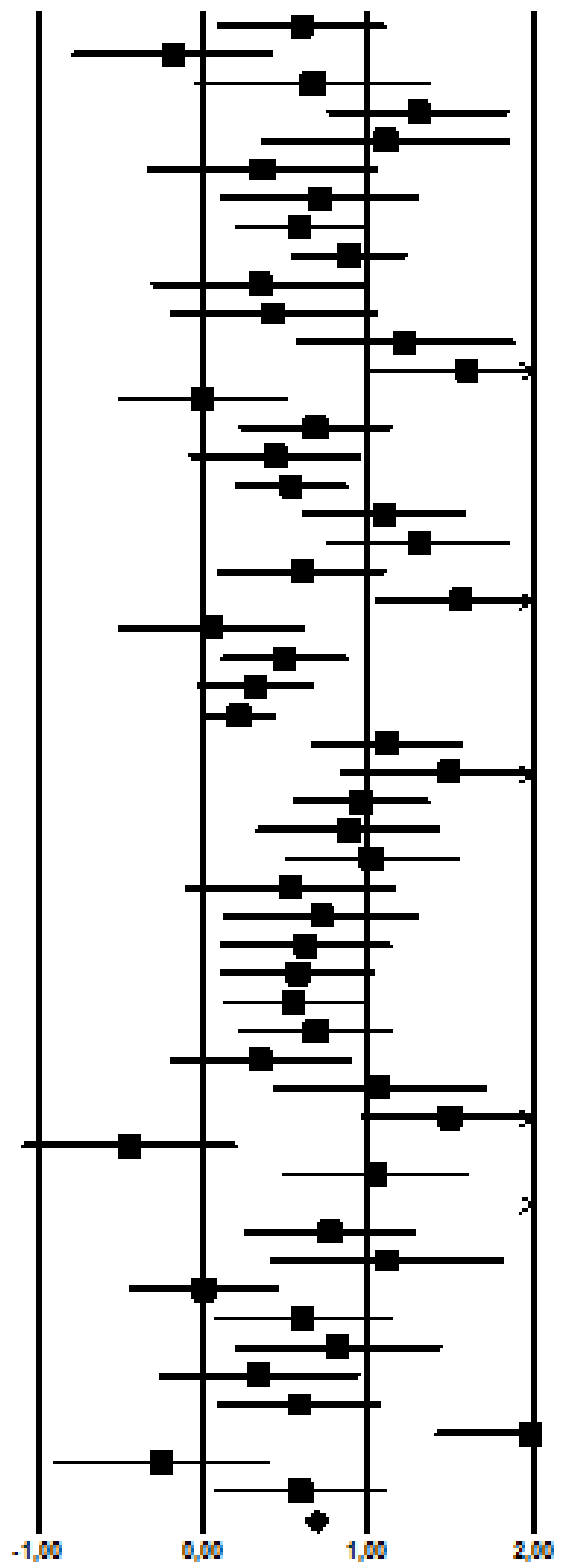

Figure 1. Effect Sizes Related to Academic Achievement in Mathematics Lesson Forest Graph 


\begin{tabular}{|c|c|c|c|c|c|}
\hline \multirow[t]{2}{*}{ atwoy name } & \multicolumn{5}{|c|}{ Etatstlos for wow studs } \\
\hline & $\begin{array}{l}\text { tha dif } \\
\text { In mons }\end{array}$ & $\begin{array}{c}\text { Gtandard } \\
\text { orror }\end{array}$ & Varane & $\begin{array}{l}\text { Lous } \\
\text { IImit }\end{array}$ & $\begin{array}{l}\text { Upper } \\
\text { Ilmit }\end{array}$ \\
\hline$\alpha_{2} w_{2}$ & 125 & 0,176 & $0,0.01$ & 095 & 134 \\
\hline EBDosums & 0.818 & 058 & 0068 & 039 & $1 \pi$ \\
\hline $\operatorname{MogA} \operatorname{mo} 7$ & 204 & $0 \mathrm{mo}$ & 0.49 & 180 & 2572 \\
\hline dark 20 & 069 & 0,25 & 0,01 & 0,12 & 124 \\
\hline HEV $/ K_{1}, N_{0}$ & 2018 & 0,313 & 0,0 & 1,64 & 28 \\
\hline $0 \alpha k \pi, 213$ & 0.98 & 0,55 & 0,05 & 0,13 & 100 \\
\hline UYGA, $\mathrm{N1}$ & $05: 4$ & 0,84 & 0,0 & -004 & 1,108 \\
\hline$\alpha \geq, 2 \omega s$ & 0,84 & 0,29 & 0,0 & 0,198 & 1,48 \\
\hline WAN, Z11 & 28 & 0,377 & 0,10 & 1,70 & 300 \\
\hline gavas & 0.44 & 0,43 & 0,069 & 0,0 & 098 \\
\hline WAG, 213 & 2196 & 0,45 & 0.24 & 1,77 & 304 \\
\hline glake, mo & 0,618 & 039 & 005 & 0013 & 12 \\
\hline GAl, $2 \mathrm{t}$ & $0 \%$ & 030 & $00 \%$ & 036 & 1,49 \\
\hline $9 k$, not & 0,813 & 030 & 0,00 & $0 m$ & 1,40 \\
\hline GTK, 201 & $0 \%$ & 031 & 0,01 & 0,007 & 1,18 \\
\hline gen $\log _{\mathrm{N}} \mathrm{x}$ & 0,718 & 0.37 & 0,142 & $-0,02$ & 1,48 \\
\hline THOSU W11 & 1005 & 0.54 & 013 & $0 \%$ & 2318 \\
\hline KNA 20 & 000 & 086 & 060 & -048 & 0,5 \\
\hline $\operatorname{MECW}, \mathrm{M}$ & 1,180 & 0,56 & 0,177 & 0,451 & $18 \pi$ \\
\hline WWWNI, $2 \mathrm{~W}$ & 0.84 & 089 & 0,67 & 0015 & 095 \\
\hline ULN M13 & 0084 & 0,35 & 0.11 & -084 & 0717 \\
\hline KANUS no & Qe日s & 0291 & 0,05 & 0,0 & $12 x$ \\
\hline EVIT 210 & 1511 & 029 & 0,0 & 0,84 & 208 \\
\hline lo $2+1$ & 1,05 & 038 & 0,114 & 0,40 & 177 \\
\hline ATN, & 10011 & 036 & 0,113 & 0,95 & $18 \pi$ \\
\hline BDW 210 & 265 & 036 & 0,18 & 1,8 & 337 \\
\hline TUT $\mathrm{No}$ & 136 & $0 \pm 0$ & 0,130 & 0,2 & 202 \\
\hline DaIR, 200 & 069 & 026 & 001 & 0,18 & 1,19 \\
\hline NAKSOS & 0,5 & 0,34 & 0,119 & $-0,17$ & $1 m$ \\
\hline Then $\mathrm{no}$ & 1284 & 03 & 0,149 & 0.88 & 20 \\
\hline YDD2, 200 & 0.84 & 037 & 0,04 & 0.13 & 1,415 \\
\hline OLOHA, TAEK, 203 & $0 \pi$ & 024 & 0,00 & 0,8 & 120 \\
\hline 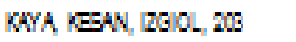 & 1,04 & 0,30 & 0,12 & 0,38 & 1,710 \\
\hline GW, OLPNA, 207 & 080 & 0,55 & 0,04 & 034 & $13 x$ \\
\hline 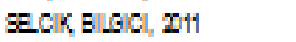 & 137 & 034 & 0,196 & 0,614 & 210 \\
\hline WA DEIR, $\mathrm{xa}$ & 0.65 & 037 & 0,04 & 0,074 & 1,178 \\
\hline BF N KTUCAGRE 207 & 0.59 & $0 \geq 3$ & 0,04 & 034 & 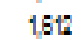 \\
\hline GWDIL EMEKBOLNATW7 & 3143 & 025 & 000 & $26 x$ & 3851 \\
\hline WA 213 & 2198 & 0.45 & 024 & 177 & 304 \\
\hline MCWR $\mathrm{x}+1$ & 084 & 0.15 & 00 & $03 \pi$ & 0974 \\
\hline ABDGEAN, $20:$ & $2 \pi 4$ & 0,819 & 03 & 1,49 & 3915 \\
\hline Googh, 2014 & 0,83 & 074 & 0,05 & 0,8 & 130 \\
\hline MeQ No & 15 & 0,30 & 008 & 0,8 & 213 \\
\hline $\mathrm{kgO}, 211$ & 297 & $0 \pi$ & 0,5 & $1,9: 4$ & 408 \\
\hline UINN, 2014 & $1+69$ & 030 & 0,111 & 0,45 & 1781 \\
\hline \multirow[t]{2}{*}{ KWOEN 2 Wh N4 } & 1,34 & 0,43 & 0,198 & 0,45 & $m$ \\
\hline & 101 & 0,043 & $0 \omega$ & 0,8 & $1,1=$ \\
\hline
\end{tabular}

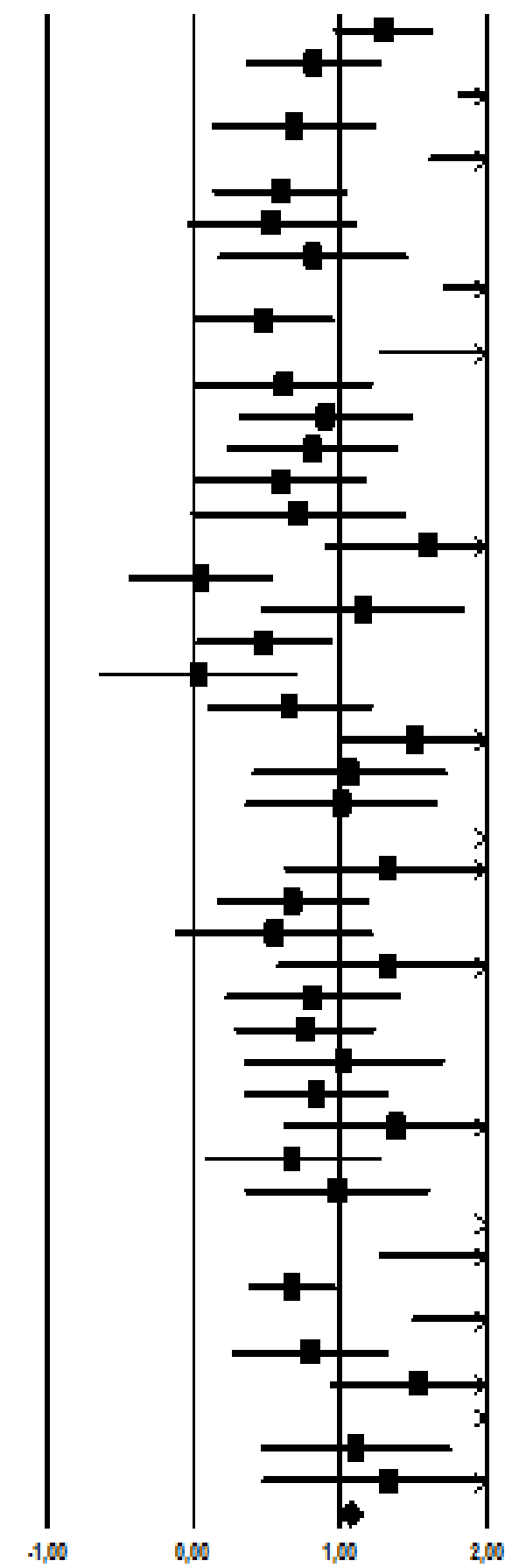

Figure 2. Effect Sizes Related to Academic Achievement in Geometry Lesson Forest Graph

When Figure 1 and Figure 2 are examined, it is seen that there is a meaningful difference in favor of TAI in terms of its effect on student achievement in both mathematics and geometry lessons. In these figures, each square shows the effect size value of the study it belongs to, and the lines extending to the right and left of each square show the $95 \%$ confidence interval for the value. The area of each square corresponds to the weight of individual studies in meta-analysis. As the sample size and precision increase, the weight of the study in meta-analysis will increase, so large squares also show studies with large samples. Finally, the diamond representation at 
the bottom indicates the overall effect size estimation obtained from meta-analysis and its confidence interval (Üstün \& Eryılmaz, 2014).

\section{Findings of the Effect Size Meta-Analysis in Terms of Academic Achievement Variable According to the Fixed Effects Model}

To find an answer to the sub-problem "Does technology supported teaching influence students' academic achievement in mathematics and geometry? " according to the fixed effect model; mean of the combined effect sizes of the general effect sizes, standard error, and the lower and upper limits according to $95 \%$ confidence interval without eliminating extreme values are shown on Table 9 below:

Table 9. Findings According to Fixed Effects Model of Effect Size Meta-Analysis in case of Academic Achievement Variable

\begin{tabular}{llllllll}
\hline Study & $\begin{array}{l}\text { Effect Size } \\
(\mathrm{d})\end{array}$ & S.E. & Variance & Lower Limit & Upper Limit & $\mathrm{Z}$ & $\mathrm{p}$ \\
\hline Mathematics & 0.687 & 0.035 & 0.001 & 0.618 & 0.756 & 19.453 & 0.000 \\
& & & & & & & \\
Geometry & 1.056 & 0.042 & 0.02 & 0.973 & 1.139 & 24.192 & 0.000 \\
\hline
\end{tabular}

As seen in Table 9, the effect size values of the studies containing data on the academic achievement are variable and combined according to the fixed effect model. The effect size value is $\mathrm{ES}=0.687$ for the mathematics lesson and the standard error of this effect size is $\mathrm{SE}=$ 0.035 while the lower and upper limit of the mean effect size is calculated as 0.618 and 0.756 , respectively. For the geometry lesson, the combined effect size value according to the fixed effects model is $\mathrm{ES}=1.056$. The standard error $\mathrm{SE}=0.042$, and the lower limit of the effect size confidence interval are 0.973 , while the upper limit is 1.139 .

According to the fixed effects model, analysis values were found to favor TAI in mathematics lesson achievement compared to traditional methods. Since the effect size value is in the range of 0.5-0.8 for mathematics achievement, it has been determined that it has a moderate effect, according to Cohen's classification (Cohen, 1988). According to Thalheimer and Cook (2002) classification, it has been found to have a moderate effect, too, since the effect size is between 0.40-0.75. When the analysis values of the studies that contain data on the academic achievement variable are analysed according to the fixed effects model in the field of geometry, a result in favor of TAI is encountered again. Since the effect size value corresponds to a value greater than 0.8 for student achievement in geometry lessons, it has a high effect, according to Cohen's classification (Cohen, 1988). According to the more detailed classification of Thalheimer and Cook (2002), it is a very high (1.10 - 1.45) level difference.

\section{Heterogeneity Test and Q-Statistics}

When the statistical significance was calculated according to the $\mathrm{Z}$ test, it was found as $\mathrm{Z}=19.449$ in the mathematics lesson area, and $\mathrm{Z}=29.914$ in the geometry lecture area while the result obtained in both branches was found to be statistically significant with $p=0.000$. It may be possible to understand the homogeneity and heterogeneity of the studies with funnel charts given in Figure 3 and Figure 4. 


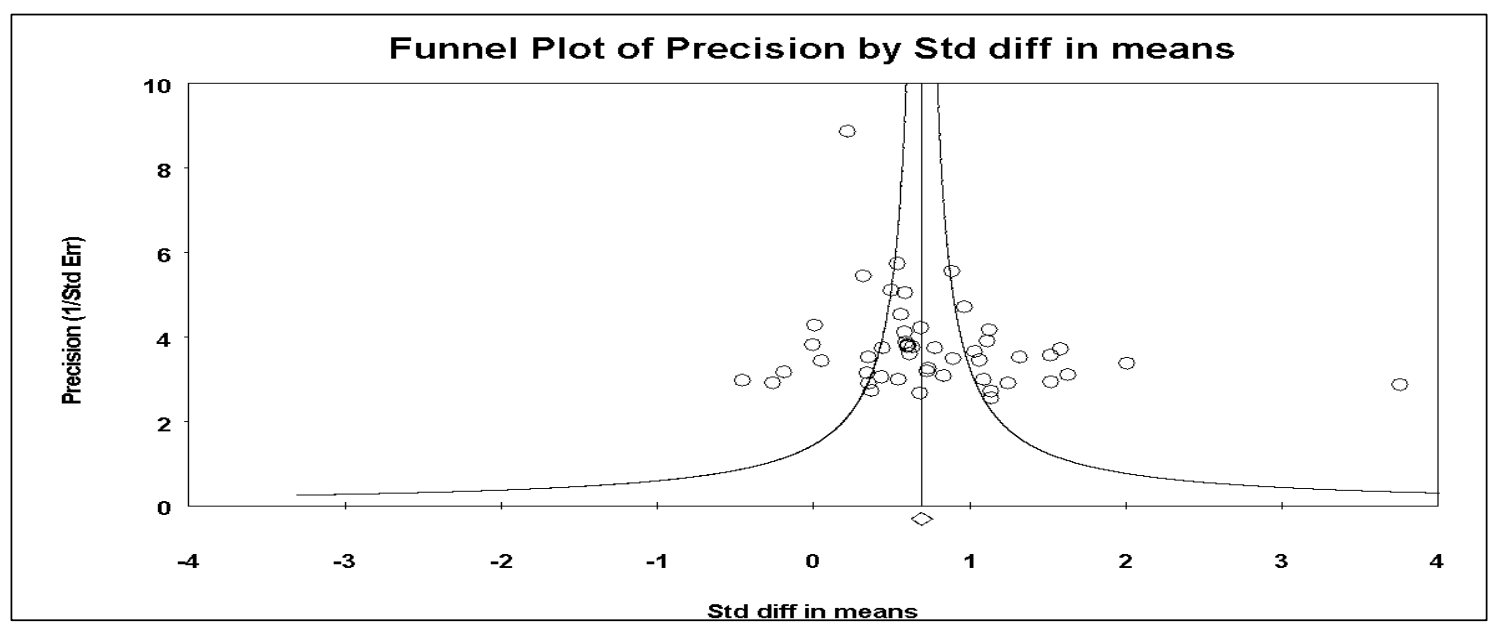

Figure 3. Funnel Plot of Studies Including Effect Size Data on Academic Achievement in Mathematics Lesson

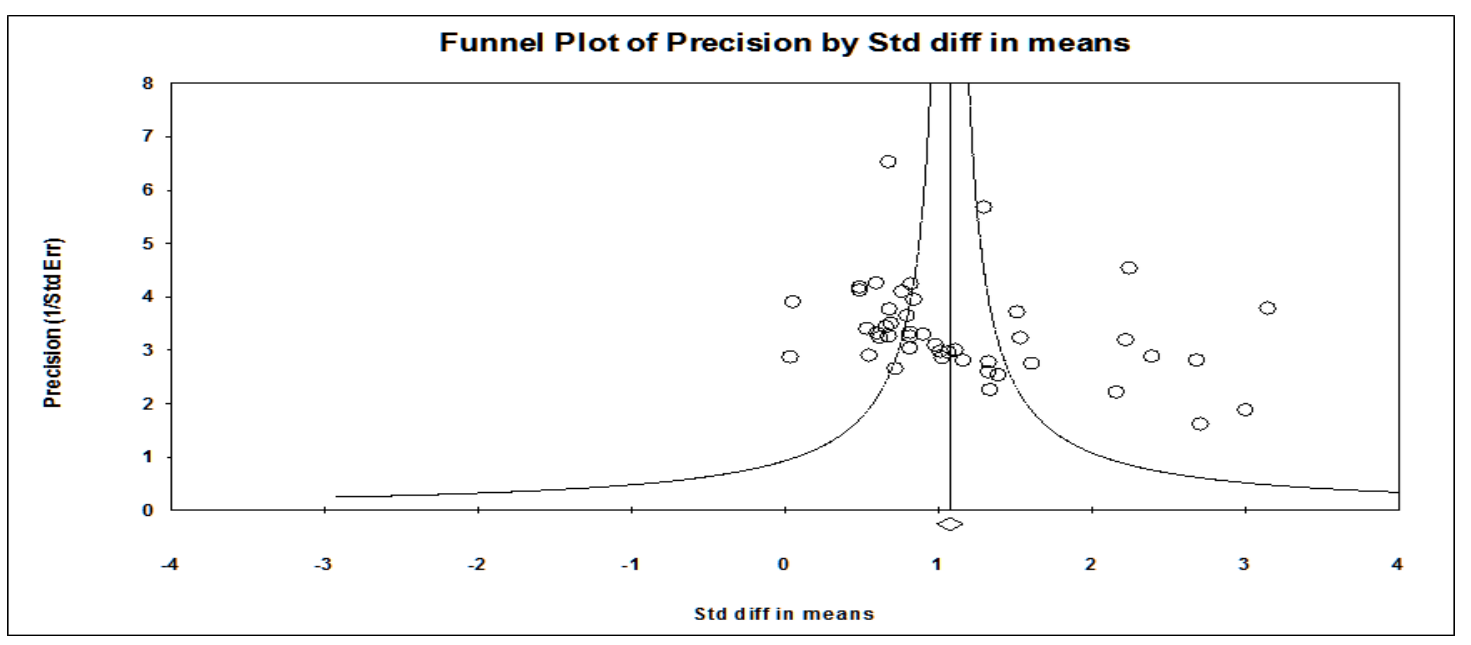

Figure 4. Funnel Plot of Studies Including Effect Size Data on Academic Achievement in Geometry Lesson

As shown in Figure 3 and Figure 4, the funnel graph is limited to \pm 1 slope. It can be interpreted that the studies are heterogeneous since almost all individual studies are not within the slope lines. However, to interpret the heterogeneity situation more precisely, the Q-statistics (homogeneity test) should definitely be examined (Dinçer, 2014). The data of the effect size values resulting from the homogeneity test are just as in Table 10.

Table 10. Homogeneity Test Results of Effect Size Distribution in Terms of Academic Achievement Variable

\begin{tabular}{llll}
\hline & Q Value & df $(\mathrm{Q})$ & $\mathrm{p}$ \\
\hline Mathematics & 236.524 & 51 & 0.000 \\
Geometry & 253.644 & 45 & 0.000 \\
\hline
\end{tabular}

When Table 10 is examined, it is seen that Q-statistics is calculated as $\mathrm{Q}=236,524$ for the mathematics. At the $95 \%$ level of significance, 51 degrees of freedom from the $\times^{2}$-table corresponds to a value of 67,505. Since the $Q$-statistic value $(Q=236,524)$ with 15 degrees of freedom is greater than the critical value of the $x^{2}$ distribution $(67,505)$, the absence hypothesis 
of homogeneity for the distribution of effect sizes was rejected in the fixed effects model. In other words, it is understood that the studies have a heterogeneous structure (Dinçer, 2014). Since the homogeneity test due to the sampling error was higher than expected, the variance of the random effect component was calculated, and the model was converted into a random effects model (K1ş, 2014). Likewise, the $\mathrm{Q}=253,644$ value found for the geometry lesson corresponds to the value of 45 degrees of freedom 61,656 from the $\times^{2}$-table at the $95 \%$ significance level. Since the $Q$-statistic value $(Q=253,644)$ is greater than the critical value of the $\times^{2} 2$ distribution with 45 degrees of freedom $(61,656)$, the absence hypothesis of homogeneity of the distribution of effect sizes is rejected in the fixed effects model, and the research model has been converted to the random effects model.

\section{Findings of Effect Size Analysis in terms of Academic Achievement Variable According to the Random Effects Model}

The average effect size is combined according to the random effects model (without removing outliers), the standard error, and the lower and upper limits according to the 95\% confidence interval are obtained from the studies included in the study according to the academic achievement variable as given.

Table 11. Findings of the Effect Size Meta-Analysis in Terms of Academic Achievement Variable According to Random Effects Model

\begin{tabular}{llllllll}
\hline Study & $\begin{array}{l}\text { Effect Size } \\
\text { (d) }\end{array}$ & $\begin{array}{l}\text { Standard } \\
\text { Error }\end{array}$ & Variance & $\begin{array}{l}\text { Lower } \\
\text { Limit }\end{array}$ & $\begin{array}{l}\text { Upper } \\
\text { Limit }\end{array}$ & Z & $\mathrm{p}$ \\
\hline Mathematics & 0.758 & 0.078 & 0.006 & 0.606 & 0.911 & 9.739 & 0.000 \\
Geometry & 1.136 & 0.103 & 0.011 & 0.935 & 1.338 & 11.055 & 0.000 \\
\hline
\end{tabular}

According to Table 11, when statistical significance was calculated according to the $\mathrm{Z}$ test, it was found as $Z=9,739$ for the mathematics lesson and $Z=11,055$ for the geometry lesson. It was determined that the analysis result has statistical significance with $p=0.000$ in both subject areas.

The combined effect size value of the studies containing data on academic achievement is variable according to the random effects model was calculated as $\mathrm{ES}=0.758$ for the mathematics lesson, and the standard error of this effect size was SE $=0.078$, the lower and upper limit of the mean effect size were calculated as 0.606 and 0.911 , respectively. Accordingly, TAI increased students' academic achievement in mathematics by 0.758 standard deviations compared to traditional teaching methods. This increase in the academic achievement of students is an indication that TAI is highly effective in the academic achievement of the mathematics course. In other words, according to the random effects model, analysis values were found to be in favor of TAI in mathematics lesson achievement compared to traditional methods. Since the effect size value is in the range of 0.5-0.8 for mathematics achievement, it has been determined that it has a moderate effect, according to Cohen's classification (Cohen, 1988). According to the classification of Thalheimer and Cook (2002), it has been found to have a high level of effect since the effect size is between 0.75-1.10.

According to the random effects model, the data collected by 46 studies included in metaanalysis within the scope of the geometry lesson; it is seen that the upper limit of 0.103 standard error and $95 \%$ confidence interval is 1.338 , and the lower limit is 0.935 , with the effect size value $\mathrm{ES}=1.136$. The geometry lesson student achievement is more positive than classical methods in favor of TAI. Because TAI increased students' achievement in geometry by 1.136 by standard deviations more than the traditional teaching methods, this is proof that TAI is very effective in the academic achievement of the geometry course. As the effect size value is greater 
than 0.8, it has been determined that it has a high effect, according to Cohen's classification (Cohen, 1988). According to the classification of Thalheimer and Cook (2002), it shows a very high level (1.10 - 1.45).

\section{Bias inside the Publications}

As Borenstein et al. (2009) defined, publication bias means that there is a tendency to publish positive and statistically significant studies when compared to studies that are negative and statistically insignificant (Kış, 2014). To see the reliability of the research, it was checked whether there is any bias in the publications. For this purpose, the publication bias of the first learning area (mathematics) is presented in Figure 5 below.

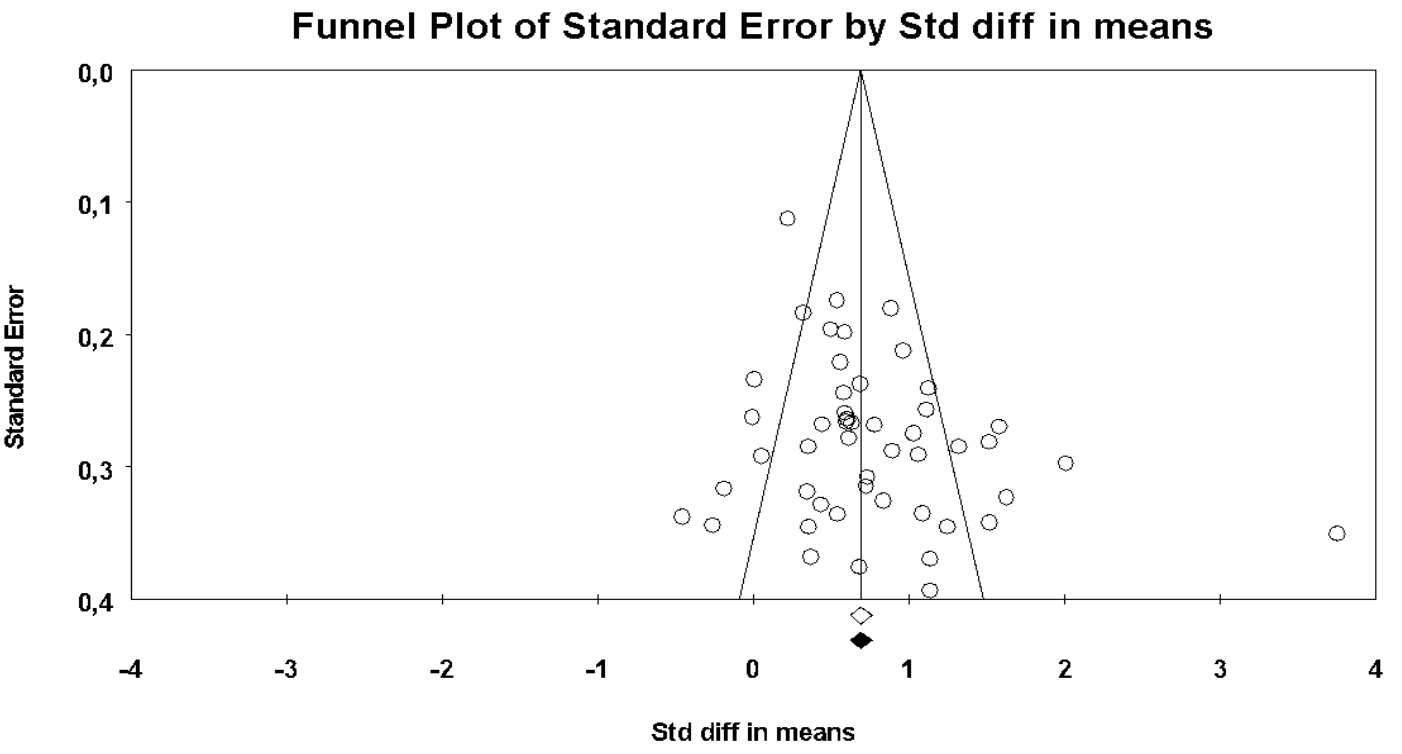

Figure 5. Funnel Plot of Studies Including Effect Size Data Related to Academic Achievement in Mathematics Lesson

In the funnel chart seen in Figure 5, the effect size is located on the horizontal (X) axis, and the standard error value is on the vertical (Y) axis. Studies with a large sample size are collected towards the top of the chart, while studies with small samples are piled up towards the bottom of the chart. Since almost all of the individual studies are located within the funnel lines symmetrically, it is understood that the studies examined do not have any publication bias (Borenstein et al., 2009). However, Egger et al. (1997) summarized the possible causes of asymmetry in the funnel plot: selection bias (publication bias, location bias), true heterogeneity, data irregularities, artifacts, and heterogeneity stemming from the wrong choice of effect size measurement, and chance alone. It is emphasized that the asymmetry in the funnel plot with the chance factor does not necessarily stem from bias (Üstün \& Ery1lmaz, 2014). Because of this, error protection number (Orwin's Fail Safe-N) was measured according to Orwin method to determine the publication bias more accurately in this study. As known, Orwin's error protection number determines the number of studies that may be missing in the meta-analysis process. (Borenstein et al., 2009).

As a result of this analysis, Orwin's Fail-Safe is calculated as N 5425. This means that the required number of studies is 5425 for the 0.758 average effect size determined as a result of the meta-analysis to reach approximately zero effect level. When the value obtained is found in the literature, the number of studies that are likely to have opposite values can invalidate the effect size obtained in the meta-analysis (Okursoy Günhan, 2009). In other words, for the 
findings of this meta-analysis, which consists of 52 studies to be considered invalid, there should be at least 5425 more studies in the literature with values opposite to the present findings. As a matter of fact, the number of studies included in meta-analysis in accordance with the inclusion criteria is 52, which can be accessed from published theses and articles about the effect of TAI on mathematics achievement. Since it is impossible to reach 5425 more studies within the specified criteria, this result is accepted as another indicator of the absence of publication bias in this meta-analysis study. Studies on the publication bias of the studies containing data on the geometry lesson area are presented below:

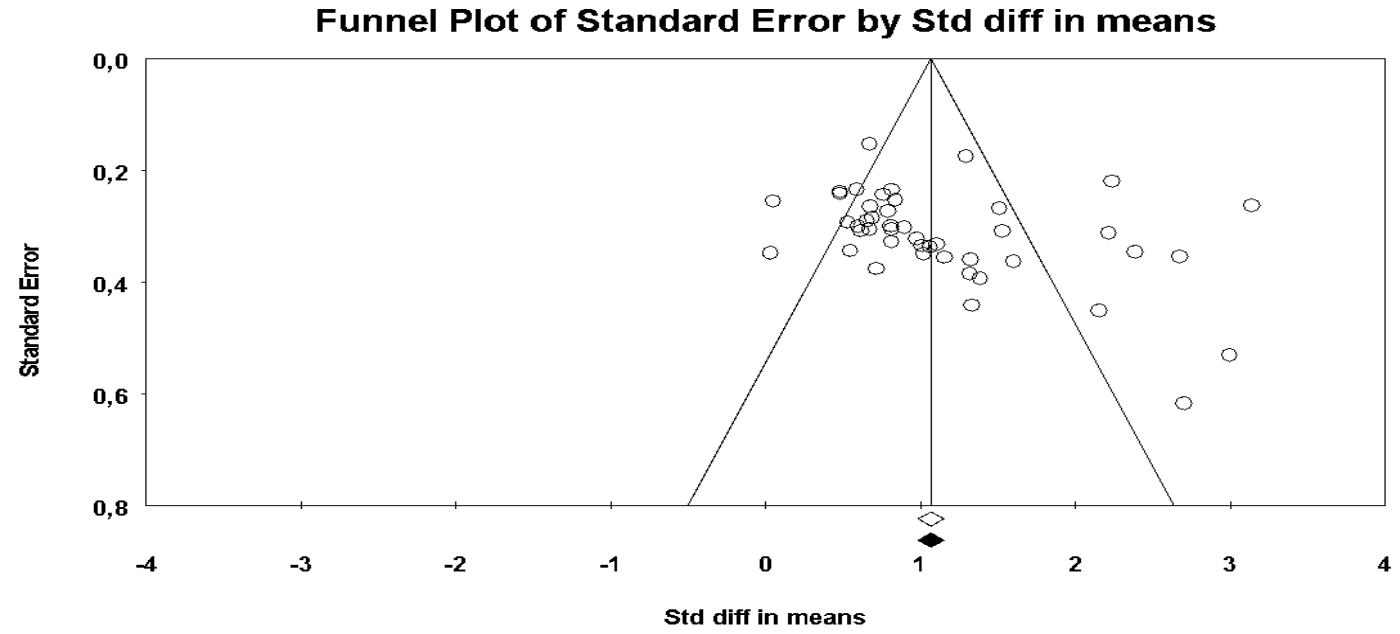

Figure 6. Funnel Scatter Plot of Studies Including Effect Size Data Related to Academic Achievement in Geometry Lesson

In Figure 6, it is seen that the majority of the 46 studies included in the study within the scope of the geometry course area are located towards the top of the figure, close to a symmetrical sequence and around the overall effect size value. In studies withno publication bias, the studies are expected to show a right and left symmetrical distribution around the vertical line representing the combined effect size. Some of the works protrude beyond the pyramid, but they still tend to accumulate in the middle and upper regions of the figure. (Borenstein et al., 2009). This graph is one of the signs that the studies do not show any publication bias. In addition, Orwin's Fail-Safe N calculation (calculation of the number of studies that may be missing in a meta-analysis) was also made to measure publication bias. Orwin's Fail-Safe was found as $\mathrm{N} 7599$ in this calculation. This result means that the required number of studies is 7599 for the effect size found as 1,136 to reach almost zero effect level. In other words, for the findings of this meta-analysis consisting of 46 studies to be considered invalid, there should be at least 7599 studies in the literature with opposite values to the present findings. However, the number of studies that are known and included in meta-analysis by the criteria determined from published theses and articles conducted in our country regarding the effect of TAI on geometry achievement is 46. Since there is no possibility to reach 7599 more studies, this value means "there is no publication bias in this meta-analysis", and there has been enough evidence to say this. 


\section{Findings Regarding the Effectiveness of Technology-Assisted Mathematics / Geometry Teaching According to the Techniques Used in the Application Process of the Studies}

The third subproblem of this meta-analysis study constitutes the question of "How does the effect sizes of the TAI techniques (animation, computer work, CD, computer software, smart board, calculator, simulation) differ?" To answer this question, the studies included in the meta-analysis were examined according to the techniques used in the application process and classified according to the titles of, interactive boards, computers, calculators, web support, and software programs. The studies' distribution according to the techniques used in the implementation process is given in Table 12.

Table 12. Frequency and Percentage Table of the Techniques Used in the Studies

\begin{tabular}{|c|c|c|c|c|c|}
\hline \multirow[t]{2}{*}{ Variable } & \multirow{2}{*}{ Technique Used } & \multicolumn{2}{|l|}{ Frequency } & \multicolumn{2}{|l|}{ Percentage } \\
\hline & & Mathematics & Geometry & Mathematics & Geometry \\
\hline & Interactive & 5 & 3 & 9.61 & 6.52 \\
\hline Academic & Computer & 15 & 7 & 25 & 13.04 \\
\hline \multirow[t]{3}{*}{ Achievement } & Calculator & 2 & 0 & 3.85 & 0 \\
\hline & applications & 20 & 36 & 42.30 & 80.43 \\
\hline & Web Supported & 10 & 0 & 19.23 & 0 \\
\hline
\end{tabular}

According to the frequency and percentage values are given in Table 12, the most frequently used technique is computer software programs in the application process of the studies examining the academic achievement variable. According to these data, it is possible to say that researchers tend to examine the effectiveness of computer applications or computer software rather than the effectiveness of interactive whiteboards, calculators, or web-assisted teaching. Especially in geometry, a very high percentage of $80.43 \%$ (academic achievement) is another data showing that the number of studies included in the meta-analysis is high, which examines software programs.

The table of effect size findings according to the techniques show that the techniques included technology support used during the implementation of the studies examined within the scope of meta-analysis is more effective, is given below:

Table 13. Effect Size Analysis Findings According to Techniques Used in Studies

\begin{tabular}{|c|c|c|c|c|}
\hline \multirow{2}{*}{ Variable } & \multirow{2}{*}{ Technique Used } & & \multicolumn{2}{|c|}{ Effect Size (d) } \\
\hline & & & Mathematics & Geometry \\
\hline \multirow{5}{*}{ Academic Achievement } & Interactive & board & 0.654 & 1.603 \\
\hline & Computer & & 0.865 & 1.213 \\
\hline & Calculator & & 0.534 & - \\
\hline & Software & applications & 0.812 & 1.094 \\
\hline & Web Supported & & 0.592 & - \\
\hline
\end{tabular}

When Table 13 is examined, according to the techniques used in the studies included in the current analysis, for the mathematics course academic achievement variable, the largest effect size was in the computer applications category with 0.865 , and the smallest effect size was in the calculator category with 0.534 . When the data were examined according to the academic achievement variable in mathematics, while interactive whiteboard, calculator, and web support had a moderate effect in Cohen's (1988) classification, computer and software applications showed a high effect. Due to the small number of studies in which calculator and interactive board applications were performed, it made the interpretation of these values difficult. Since it was useful to give an idea.

According to the academic achievement variable of the geometry lesson, it can be said that there is a high effective on the data in all applications (Cohen, 1988). According to the 
classification of Thalheimer and Cook (2002), while the interactive board has an excellent effect with an effect coefficient of $1.603(1.45<\mathrm{d})$, computer and software applications show a very high effect $(1.10-1.45)$. Generally speaking, it is possible to say that the technique that increases student achievement is computer applications and software programs.

\section{Findings Regarding the Effectiveness of Technology-Assisted Instruction According to the Learning Area (Mathematics / Geometry) Taught in Studies}

"When examined in terms of course areas (mathematics and geometry) where the studies are carried out, what kind of difference is there between the effect sizes of the TAI method on academic achievement?" To find an answer to this sub-problem, the general effect size values obtained according to the learning areas in the studies included in the analysis were compared. The data obtained regarding these findings are given in Table 14.

Table 14. General Effect Size Analysis Findings According to the Learning Areas in which the Studies were Conducted.

\begin{tabular}{ll}
\hline Learning Area & Effect Size Value $(\mathrm{d})$ \\
& Academic Achievement \\
\hline Mathematics & 0,758 \\
Geometry & 1,136 \\
\hline
\end{tabular}

As can be seen in Table 14, the effect size of TAI on students' achievement in mathematics and geometry was found to be 0.758 and 1.136 , respectively. While the result of 0.758 calculated as the effect size has a moderate effect according to Cohen's (1988) classification, the value of 1.136 indicates a high level of effect size. According to Thalheimer and Cook (2002), while TAI has a high effect on the academic achievement of the mathematics course, it has a very high effect on the geometry lesson. In other words, TAI increases students' achievement in geometry more than it does in mathematics, compared to traditional teaching methods.

\section{Findings Regarding the Effectiveness of Technology-Assisted Mathematics/ Geometry Teaching According to the Education Level of the Study Samples}

The fourth sub-problem of the study was: "Is there a difference between the effect sizes of technology-supported teaching methods in terms of students' education level (preschool, primary school, middle school, high school, university)?". To find an answer to this subproblem, the studies examined in the study were categorized according to the academic achievement variable in mathematics and geometry lessons and analyses were made on the relevant data. The distribution of the studies according to the education levels dealt with is given in Table 15 below:

Table 15. Frequency and Percentage Table Regarding the Education Levels where the Studies were Conducted.

\begin{tabular}{llllll}
\hline & \multirow{2}{*}{ Teaching Level } & Frequency & \multicolumn{3}{c}{ Percentage } \\
\cline { 3 - 6 } & Mathematics & Geometry & Mathematics & Geometry \\
\hline \multirow{3}{*}{ Academic } & Preschool & 1 & 1 & 1.92 & 2.17 \\
Achievement & Elementary & 4 & 2 & 7.69 & 4.35 \\
& Middle School & 24 & 32 & 46.15 & 69.57 \\
& High School & 12 & 8 & 23.08 & 17.39 \\
& University & 11 & 3 & 21.15 & 6.52 \\
& (Undergraduate) & & & & \\
\hline
\end{tabular}

According to Table 15, while studies on the effectiveness of TAI in terms of academic achievement at secondary school (5th, 6th, 7th, and 8th grade) in both subject areas constitute most of the studies $(57.14 \%)$ included in meta-analysis. In the study, it is seen that the number of studies $(2.04 \%)$ implemented at the preschool level is quite low. Table 16, which shows the 
comparison of the general effect sizes of the samples in terms of education levels according to the academic achievement variable, is shown below. As a result of the homogeneity tests conducted based on the teaching level, it was determined that the studies at all levels have a heterogeneous structure. For this reason, the values in Table 16 are given according to the random-effects model.

Table 16. Effect Size Analysis Findings of Study Samples Regarding Education Level According to Academic Achievement Variable

\begin{tabular}{lll}
\hline \multirow{2}{*}{ Teaching Level } & Effect Size $(\mathrm{d})$ & \\
\cline { 2 - 3 } & Mathematics & Geometry \\
\hline Preschool & 1.554 & 2.916 \\
Elementary & 0.567 & 0.959 \\
Middle School & 0.719 & 0.994 \\
High School & 0.776 & 1.769 \\
University (Undergraduate) & 0.845 & 0.852 \\
\hline
\end{tabular}

When Table 16 is examined, it is seen that the highest effect value in the field of mathematics belongs to the preschool level with a value of $d=1.554$. However, since this result belongs to $1(\mathrm{~N}=1)$ study, it cannot be said to be a significant finding. Similarly, since the number of studies in the geometry lesson preschool academic achievement group is 1, it can be said that the values found only reveal the current situation. Although there is a moderate effect $(0.5-$ 0.8), according to Cohen's (1988) classification in primary, secondary, and high school levels, the university level has a high effect $(0.8<\mathrm{d})$ with an effect value of 0.858 . According to the detailed classification of Thalheimer and Cook (2002), since the effect value of studies with primary and secondary school samples is between $0.40-0.75$, the effect size of studies with a medium level effect, high school and university level samples is $0.75-1$, since it is in the range of 10, it has a high level of impact. Based on these results, it can be argued that the higher the teaching level, the higher the mathematics achievement scores.

According to Table 16, when the effect sizes of the education levels in geometry are examined, it is seen that all levels (preschool, primary school, secondary school, high school, university) have a high level of effect since the values of Cohen's (1988) classification are higher than 0.8. According to the detailed classification of Thalheimer and Cook (2002), the effect sizes of the studies that have samples at the primary and university levels are in the range of $0.75-1.10$, while the studies at the secondary school level have an effective value in the range of 1.10 1.45, it shows a very high level of effect. Although preschool level has the highest effect value, this finding cannot be significant since the number of studies having data at preschool level is $1(\mathrm{~N}=1)$. According to the academic achievement variable, the level that has an excellent is studied containing data belonging to the high school level with an effective value greater than 1.45 (Thalheimer \& Cook, 2002). It was also found that the effect of TAI on students' achievement in geometry was greater than its effect on mathematics. This can be interpreted as TAI improves students' geometry achievement more than mathematics achievement.

In summary, within the direction of the analysis results of Table 16, it can be said that because the effect size obtained according to the academic achievement variable is higher in the geometry lessons, it can be said that TAI has a more positive effect on the academic achievement of geometry compared to the mathematics lessons. In addition, the undergraduate (university) level has the highest effect $(\mathrm{d}=0.845)$ in mathematics, while the secondary school level has the highest effect $(d=0.994)$ in geometry. 


\section{Conclusion and Recommendations}

In this title, based on the results obtained regarding the main findings of the analysis study and the results given in parallel with the sub-problems of this research, some suggestions were made below.

\section{Conclusions}

In this study, it was aimed to determine the effect of TAI on the academic achievement of students in mathematics and geometry courses, and the experimental studies conducted on the subject were examined according to the inclusion criteria, and their findings were combined with a meta-analytical method.

\section{Results Regarding the Academic Achievement Variable}

To calculate the TAI's effect size of students' academic achievements in the mathematics and geometry lessons, 52 studies in accordance with the criteria determined were examined within the scope of mathematics lessons, and 46 studies within the scope of geometry lessons. The number of research increased after 2007, the master's thesis $(n=70,71.43 \%)$ in terms of the type of publications, the secondary school level $(n=56,51.14 \%)$ on the basis of the education level where the studies were carried out, software programs according to the technique used in the application process $(n=56,51.14 \%)$ ranked first.

When the effect size results of the studies included in meta-analysis are examined according to academic achievement variable; it was observed that $48(92.3 \%)$ studies in the mathematics lesson and $46(100 \%)$ studies in geometry lesson had positive effect size, and 4 (7.69\%) studies in mathematics lesson had negative effect size. The fact that almost all the studies had a positive effect size shows that the academic achievement value in these studies favors the experimental group, depending on the degree of effect size. When the effect sizes are classified according to Cohen's (1988) classification, it was found that 9 studies $(17.30 \%)$ were small, 17 studies (32.69\%) were medium, and 20 studies $(38.46 \%)$ had a large effect on the academic achievement of mathematics. Looking at the geometry lesson findings 2 studies $(4.35 \%)$ were small, 14 studies $(30.43 \%)$ were medium, and 28 studies $(60.86 \%)$ had a high effect.

As a result of the combination according to the fixed effects model, it was seen that there was a positive and statistically significant $(\mathrm{p}<.05)$ effect size of 0.687 for the mathematics lesson and 1.056 for the geometry lesson in favor of TAI. This finding is a moderate value according to the classification of Cohen (1988), and Thalheimer and Cook (2002).

The heterogeneity test was performed for the model selection to be used to calculate the overall effect, and the Q value was calculated as 236.524 in the mathematics course area. At the $95 \%$ significance level, 51 degrees of freedom value from the $\times \wedge 2=$ table corresponds to the value of 67,505. Since the $Q$-statistic value $(Q=236,524)$ with 51 degrees of freedom is greater than the critical value of the $\times^{\wedge} 2$ distribution $(67,505)$, it has been concluded that the studies are heterogeneous. The same is true for geometry. For this reason, the random effects model was used as the analysis model, and the overall effect size was calculated as 0.758 within the boundaries of 0.606 and 0.911 for mathematics and 1.336 within the limits of 0.935 and 1.338 for geometry. It was determined that the analysis result has statistical significance with $\mathrm{p}=$ 0.000 in both subject areas. it is stated that TAI increases the mathematics academic achievement score by approximately 0.76 and geometry by approximately 1.34 standard deviation. The fact that students' academic achievement has increased so much is an indication that TAI is very effective in students' achievement in mathematics and geometry lessons. According to Cohen's (1988) classification, this result is a medium level but close to a high level in the mathematics lesson and a high-level result in the geometry lesson. According to the 
classification of Thalheimer and Cook (2002), TAI shows a high level of effect on student achievement for mathematics and a very high effect on geometry lessons.

In addition, it can be said that TAI in the geometry lesson area has a more positive effect on academic achievement than the mathematics lesson due to the greater effect size obtained in the studies containing data on the geometry.

The publication bias of the studies included in the study according to the academic achievement variable was tested with both Orwin's Fail-Safe N account and funnel scatter plot. It was found that there was no publication bias in terms of the academic achievement variable of the study by examining and interpreting it with funnel scatter plot and Orwin's Fail-Safe N method for both areas.

\section{Results According to the Techniques Used in the Application Process of the Studies}

Technology-supported techniques used by the studies included in the meta-analysis during the application process were examined. it was found that there were 5 categories: interactive whiteboard, computer, calculator, software programs, and web support. Among these categories, software programs have the highest value, with a $55.86 \%$ share. Especially in geometry, $80.43 \%$ (academic achievement) showed that the number of studies examining software programs among the data included in the current research with a very high percentage. However, another remarkable point is that the number of studies examining the effectiveness of the calculator $(\mathrm{N}=2,3.85 \%)$ is quite low.

It is the technical computer applications that TAI has the greatest effect on the academic achievement for the mathematics course. The result of this effect size, which is found to be high, positive, and statistically significant, is 0.865 (Cohen, 1988). The second highest impact factor belongs to software programs $(\mathrm{d}=0.812)$. While the calculator has a low impact on the mathematics achievement variable, the interactive whiteboard and web support applications have a moderate impact. On the other hand, all techniques (interactive whiteboard, computer, and software programs) have a high level of influence on the academic achievement for the geometry course. Generally speaking, it is possible to say that the techniques that increase student achievement relatively more are computer applications and software programs.

\section{Results According to the Teaching Areas of the Studies}

Positive and significant results were obtained regarding the achievement variable in both course areas (mathematics / geometry). The effect size of TAI on students' achievement in mathematics and geometry courses was found as 0.758 (medium level) and 1.136 (high level), respectively. As a result, TAI increases students' achievement in geometry lessons more than their achievement in mathematics lessons than traditional teaching methods.

\section{Results According to the Education Level of the Study Samples}

The findings reached by the study is conducted of the studies included in the analysis stage review of studies on the effects on academic achievement in secondary level TAI mathematics and geometry courses in Turkey has created a 55,17\% volume of the studies included in the meta-analysis. While it was found that the studies conducted at the secondary school level were more common, the number of studies conducted at the preschool level $(2.04 \%)$ was found to be quite low.

The TAI method was most effective at the university level $(\mathrm{d}=0.858)$ in the academic achievement for mathematics, and it has a moderate effect in primary, secondary, and high school levels, according to Cohen's (1988) classification. As the education level increased, the mathematics achievement scores also increased. When the effect sizes of education levels in geometry lesson areas are examined, it is seen that the values of all levels (preschool, primary 
school, middle school, high school, university) have a high level of effect. It has also been concluded that the effect of TAI on students' achievement in geometry has more effect size than its effect on mathematics achievement; thus, it has been concluded that TAI improves students' geometry achievement more than their mathematics achievement. In addition, while the undergraduate level has the highest effect $(\mathrm{d}=0.845)$ in mathematics, geometry has the highest effect $(\mathrm{d}=0.994)$ at the secondary school level.

\section{Suggestions}

Based on the results of the study, the following recommendations can be made for the researchers and practitioners.

\section{Suggestions for Researchers}

According to the findings of the study, TAI has a greater effect value on the academic achievement variable in the geometry course area compared to the mathematics course area. The reason for this difference, which varies according to the course area, can be investigated.

In the current meta-analysis study, the achievement of TAI in mathematics and geometry and its effectiveness in these lessons were examined and the effects other than these were excluded from the scope of the study. Again, the general effect of the studies that examine the effect of TAI on variables such as self-efficacy perception, problem solving skill, permanence, spatial thinking, and anxiety can be investigated by doing meta-analysis.

It was determined that more than half of the studies included in the analysis were conducted at the secondary school level. The increase in the number of studies with pre-school and undergraduate level samples, where the number of studies is quite low, will be more decisive in terms of seeing the general picture.

This study sample was composed of only the studies conducted in Turkey. The opportunity to compare can be provided by making an international sample of the same study. Only studies with quantitative data were used in the study. As a complement to this research, more comprehensive results can be obtained by examining qualitative studies in the subject area.

\section{Suggestions for Practitioners}

Although there are many investigations and a wide range of resources in the field of meta-analysis, which is the method of the research, there is very limited research and resources in our country. In addition, the need for meta-analysis, which brings common results by combining similar study data, which is increasing day by day in the literature, reveals the need for more studies on this subject. For this purpose, researchers can be supported, and postgraduate meta-analysis courses can be included.

The meta-analysis method is a study that requires systematic and planned study. Missing even one of the variables to be used as data requires a re-examination of all studies. In this respect, researchers considering meta-analysis should make an excellent plan initially and work very carefully during the coding of the data.

One of the difficulties of the meta-analysis method is that the studies comply with the criteria determined to answer the question to be investigated in the meta-analysis. At this point, while coding studies for calculating the effect size, studies that do not have the necessary numerical data pose difficulties. In this case, existing data are calculated with statistical methods, or researchers are tried to be reached. Studies whose data are not available cannot be included in the meta-analysis. To solve such difficulties, a standard can be provided for presenting data from studies. In this way, the coding of the studies included in the meta-analysis will be more 
systematic and easier, and more comprehensive and healthy results will be achieved.

Publication bias is one of the main problems in terms of the validity for the meta-analysis review. To eliminate publication bias, it is crucial to include published and unpublished studies in the analysis. However, reaching unpublished studies is one of the research difficulties. In this context, the relevant units may be recommended to create a common sharing platform by removing the obstacles to sharing scientific knowledge.

\section{Note}

This article is produced from the Serap DENIZ's master thesis.

\section{References}

Arslan, A. (2008). Web destekli öğretimin ve öğretimsel materyal kullanımının öğrencilerin matematik kaygisına, tutumuna ve başarısına etkisi [The effects of web supported instruction and use of instructional materials on students? Mathematics anxieties, attitudes, and achievements]. Marmara Üniversitesi [Marmara University]: Doktora tezi [Doctoral thesis].

Baki, A. (2002). Bilgisayar destekli matematik ögretimi [Computer aided mathematics teaching]. İstanbul: TÜBİTAK/BİTAV Ceren Yayınları [TÜBİTAK/BİTAV Ceren Pub.].

Binbay, T., Ulaş, H., Elbi, H., \& Alptekin, K. (2011). Türkiye' de psikoz epidemiyolojisi: Yaygınlık tahminleri ve başvuru oranları üzerine sistematik bir gözden geçirme [The psychosis epidemiology in Turkey: A systematic review on prevalence estimates and admission rates]. Türk Psikiyatri Dergisi [Turkish Journal of Psychiatry], 22(1), 40-52.

Borenstein, M., Hedges, L., Higgins, J. P. T, \& Rothstein, H. (2009). Meta-analysis fixed effects vs. random effects. United Kingdom:_John Wiley \& Sons

Camnalbur, M. (2008). Bilgisayar destekli öğretimin etkililiği üzerine bir meta analiz çalışması [A meta-analysis for the effectiveness of computer based education]. Marmara Üniversitesi [Marmara University]: Yüksek Lisans Tezi [Master's thesis].

Carter, V., \& Good, E. (1973). Dictionary of Education, 4th Edition. New York: McGraw Hill Book Company.

Cohen, J. (1988). Statistical power analysis for the behavioral sciences (2nd ed.). Hillsdale, NJ: Lawrence Earlbaum Associates.

Cohen, L., Manion, L., \& Morrison, K. (2007). Research methods in education, 6th edition. Rotledge Falmer, New York.

Çavuş, H. (2006). Türkiye'de matematik öğretiminde öğretmenlerin ĕgitim ortamlarında bilgisayar ve matematik programlarından yararlanma düzeyleri [Mathematics teachers' usage level of the computer and mathematics programs at the educational contexts in Turkey]. Yüzüncü Y1l Üniversitesi [Yuzuncu Yil University]: Doktora tezi [Doctoral thesis].

Dinçer, S. (2014). Eğitim bilimlerinde uygulamall meta-analiz [Applied meta-analysis in educational sciences]. Ankara: Pegem Akademi Yayınları [Pegem Academy].

Durmuş, S. (2001). Matematik eğitimine oluşturmacı yaklaşımlar [Constructivist approaches to mathematics education]. Kuram ve Uygulamada Ĕgitim Bilimleri [Educational Sciences: Theory and Practice], 1, 93-107.

Egger M, Smith G. D., \& Phillips A.N., (1997). Meta-analysis: Principles and procedures. the BMJ, 315, 1533-1537.

Eğitimi Araştırma ve Geliştirme Dairesi. (2016). Eğitim teknolojisi kllavuzu [Education technology guide]. Ankara: MEB EARGED Yayınları [MEB EARGED Pub.]. 
Ersoy, Y. (2003). Teknoloji destekli matematik öğretimi- II: hesap makinesinin matematik etkinliklerinde kullanılması [Technology supported mathematics teaching-ii: using calculator in mathematics activities]. Illkögretim-online [Elementary Education Online], 2(2), 19-27. http://ilkogretim-online.org.tr/

Ersoy, Y. (2003). Teknoloji destekli matematik egitimi-1: Gelismeler, politikalar ve stratejiler. [Technology supported mathematics education-1: Developments, policies and strategies]. Elementary Education Online, 2(1), 18-27.

Ersoy, Y. (2005). Matematik eğitimini yenileme yönünde ileri hareketler-I: Teknoloji destekli matematik öğretimi [Movements for innovations of mathematics education-I: Technology supported mathematics teaching]. The Turkish Online Journal of Educational Technology, 4(2), 51-63.

Ersoy, Y. (2005). Fen lisesi matematik öğretmenlerinin görüşleri-II: Matematik öğretim ortamı ve baz1 kisitlar [The views of science lyceum mathematics teachers-II: The media of mathematics teaching and some constraints]. The Turkish Journal of Educational Technology, 4(4). 135-145.

Ertem Akbaş, E. (2016). Meslek yüksekokulu ögrrencilerinin bilgisayar destekli ortamda "limitsüreklilik" konusundaki ögrenmelerinin solo taksonomisine göre değerlendirilmesi [Evaluation of the learning of the students of vocational high schools in computer assisted environment about the concept of 'limit and continuity' by SOLO taxonomy]. Karadeniz Teknik Üniversitesi [Karadeniz Technical University]: Doktora tezi [Doctoral thesis].

Ertem Akbaş, E. (2019). The impact of EBA (educational informatics network) assisted mathematics teaching in 5th grade fractions on students' achievements. Journal of Computer and Education Research Year, 7(13), 120-145.

Güven, B. (2002). Dinamik geometri yazılımı cabri ile keşfederek geometri ögrenme [Exploratory geometry learning within cabri-based environment]. Karadeniz Teknik Üniversitesi [Karadeniz Technical University]: Yüksek Lisans Tezi [Master's thesis].

Hacısalihoğlu Karadeniz, M., \& Akar, Ü. (2014). Dinamik geometri yazılımının açıortay ve kenarortay öğretiminde meslek lisesi öğrencilerinin başarılarına etkisi [The effect of dynamic geometry software on the vocational high school students' succes for teaching bisector and the median concepts]. Bilgisayar ve Eğitim Araştırmaları Dergisi [Journal of Computer and Education Research], 2(4), 74-90.

Karal, H., \& Berigel, D. S. (April, 2008). Matematik ögretmenlerinin 3 boyutlu kavramlar ögretmede yaşadıkları sorunlara bilgisayar destekli bir çözüm önerisi [A Computer aided solution for mathematics teacher's problems teaching 3 dimensional concepts]. 2. Uluslararası Bilgisayar ve Ögretim Teknolojileri Sempozyumu [2nd International Computer and Instructional Technologies Symposium] (pp.666-672). Izmir, Turkey.

Kış, A. (2014). Okul müdürlerinin ögretimsel liderlik davranışlarını gösterme düzeylerine ilişkin yönetici ve ögretmen görüşlerine yönelik bir meta-analiz. [The views of administrators and teachers on levels of principals instructional leadership behaviours: A meta-analysis]. İnönü Üniversitesi [Inonu University]: Yüksek Lisans Tezi [Master's thesis].

Milli Eğitim Bakanlığı (2016). İlköğretim matematik dersi öğretim programı ve kllavuzu [Elementary math curriculum and guide]. Ankara. Ankara: MEB Yayınları [MEB Pub].

Milli Eğitim Bakanlığı. (2016). Ortaöğretim matematik dersi (9, 10, 11 ve 12. sinıflar) ögretim programı [Secondary education mathematics course (grades 9, 10, 11 and 12) curriculum] Ankara: MEB Yayınları [MEB Pub].

National Council of teachers of Mathematics (2004). Principles and standards for school mathematics. VA: Philadelphia.

Nikolaou, C. (2000). Hand-held calculator use and achievement in mathematics education: A meta-analysis. Georgia State University: Doctoral thesis. 
Okursoy Günhan, F. (2009). Kavram haritaları öğretim stratejisinin öğrenci başarısına etkisi: bir meta analiz çalışması [The effect of concept mapping on learner success: A metaanalytic study]. Marmara Üniversitesi [Marmara University]: Yüksek Lisans Tezi [Master's thesis].

Olkun, S., \& Toluk, Z. (2001). Illköğretimde matematik ögretimi 1 - 5 sinıflar [Teaching mathematics in primary education grades 1-5]. Ankara: Artım Yayınlar1 [Artım Pub].

Öksüz, C., \& Ak, Ş. (2010). İlköğretim okullarında matematik derslerinde teknoloji kullanım düzeyini belirleme ölçeği geçerlik ve güvenirlik çalışması [A validity and reliability study of the level of technology use scale in mathematics lessons at elementary school level]. Elektronik Sosyal Bilimler Dergisi [Electronic Journal of Social Sciences], 9(32), 372-383.

Özcan, Ş. (2008). Ĕgitim yöneticisinin cinsiyet ve hizmet içi eğitim durumunun göreve etkisi: Bir meta analitik etki analizi [The effect of education managers' gender and in-service training status on the task: A meta-analytical impact analysis]. Marmara Üniversitesi [Marmara University]: Doktora Tezi [Doctoral thesis].

Özkeleş-Çağlayan, S. (2010). Lise 1. sınıf öğrencilerinin geometri dersine yönelik özyeterlik algisl ve tutumunun geometri dersi akademik başarısinı yordama gücü [The potential of predictive of the academic achievement points of geometry using the 9th grade students geometry self-efficacy and geometry attitude]. Y1ldız Teknik Üniversitesi: Yüksek Lisans Tezi [Master's thesis].

Tarım, K. (2003). Kubaşık öğrenme yönteminin matematik öğretimindeki etkinliği ve kubaşık öğrenme yöntemine ilişkin bir meta analiz çalışmasl [Effectiveness of cooperative learning method on teaching mathematics and meta analytic study for cooperative learning method]. Çukurova Üniversitesi [Cukurova University]: Doktora Tezi [Doctoral thesis].

Thalheimer, W., \& Cook, S. (2002, August). How to calculate effect sizes from published research articles: A simplified methodology. Retrieved November 31, 2002, from http://work-learning.com/effect_sizes.htm.

Üstün, U., \& Eryılmaz, A. (2014). Etkili araştırma sentezleri yapabilmek için bir araştırma yöntemi: Meta-analiz [A research methodology to conduct effective research syntheses: Meta-analysis]. Education and Science, 39(174), 1-32.

Wolf, F. M. (1986). Meta-analysis: Quantitative methods for research synthesis. California: Sage Publications Inc.

Yıldız, N. (2009). Meta-analizinde heterojenliğin ve farklı varyans tahmin yöntemlerinin incelenmesi [Analysis of the heterogeneity and various variance estimation methods in meta-analysis]. Marmara Üniversitesi [Marmara University]: Doktora Tezi [Doctoral thesis]. 


\section{ANNEXS}

\section{ANNEX 1: Meta Analysis Coding Form}

I. Research ID

1. Research Name

2. Author Name/s

3. Research Year

4. Application City

5. Publication of the Research

II. Content of the Research

1. Learning Area (mathematic /geometry)

2. Subject of Application

3. Education Level

4. The type of technology used.

5. Application Period.

6. Dependent Variables (success, attitude)

III. Research Data.

1. Number of Samples $(\mathrm{N})$

2. Mean $(X)$

3. Standard Deviation (SD)

\section{ANNEX 2: Studies Included in Meta-Analysis.}

Abdüsselam, M. (2006). Matematiksel denklem ve ifadelerin bilgisayar ortaminda grafikleştirilerek ögretilmesinin eğitime katkular [The contribution of teaching mathematical equation and expressions by using computer graphics to education]. Karadeniz Teknik Üniversitesi [Karadeniz Technical University]: Yüksek Lisans Tezi [Master's thesis].

Ağaç, G. (2009). Lise ögrencilerinin trigonometri öğrenme alanında grafik hesap makinesi kullanımının akademik başarlya ve problem çözme becerisine etkisi [Effect of graphic calculating machine utilization by high school students in learning trigonometry on their academic achievements and problem-solving skills] Dokuz Eylul University: Yüksek Lisans Tezi [Master's thesis].

Akçayır, M. (2011). Akıllı tahta kullanılarak işlenen matematik dersinin sınıf öğretmenliği birinci sını ögrencilerinin başarl, tutum ve motivasyonlarına etkisi [The effect of using interactive whiteboard in mathematic lesson upon students? motivation, academic success, and attitudes]. Gazi University: Yüksek Lisans Tezi [Master's thesis].

Aktümen, M. (2002). Ilkögrretim 8. Sinıflarda harfli ifadelerle işlemlerin öğretiminde bilgisayar destekli ögretimin rolü [The role of computer assisted instruction in the teaching of expressions among primary education eight grade students].

Aktümen, M., Kaçar, A. (2008). Bilgisayar cebiri sistemlerinin matematiğe yönelik tutuma etkisi.] Gazi University: Yüksek Lisans Tezi [Master's thesis].

Aksoy, Y. (2007). Türev kavramının ögretiminde bilgisayar cebiri sistemlerinin etkisi [The effect of computer algebra systems on the teaching of derivative concept] Gazi University: Doktora Tezi [Doctoral thesis].

Aksoy, N., C., Çalık, N., \& Çinar, C. (Haziran, 2012). Excel ile matematik öğretiminin ögretmen adaylarının fonksiyon grafikleri çizimi üzerine etkisi [The effect of teaching mathematics with Excel on pre-service teachers' drawing of function graphs]. X. Ulusal 
Fen Bilimleri ve Matematik Eğitimi Kongresi [X. National Science and Mathematics Education Congress.]

Aksoy, N. C. (2014). 9. Dijital oyun tabanlı matematik öğretiminin ortaokul 6. Sınıf öğrencilerinin başarılarına, başarı güdüsü, öz-yeterlik ve tutum özelliklerine etkisi [Effects of digital game-based mathematics teaching on 6th grades students' achievement, motivation, attitude, and self-efficacy]. Gazi University: Doktora Tezi [Doctoral thesis].

Alabay, E. (2006). Altı yaş okulöncesi dönemi çocuklarına bilgisayar destekli matematiksel kavramların ögretimi [Make to learn the children who are attending the pre-school age of six about same mathematical concepts with the helping of computer]. Selcuk University: Yüksek Lisans Tezi [Master's thesis].

Altın, S. (2012). Bilgisayar destekli dönüşüm geometrisi öğretiminin 8. sınıf ögrencilerinin başarisina ve matematik dersine yönelik tutumuna etkisi [The effect of computer aided transformation geometry instruction on 8th grade students? mathematics succes and attitude]. Eskişehir Osman Gazi University: Yüksek Lisans Tezi [Master's thesis].

Andiç, T. (2012). İlköğretim 8. Sinıf matematik dersi permütasyon kombinasyon konusunun bilgisiyar destekli ögretiminin ögrenci erişi düzeylerine ve tutumlarına etkisi [The effect of computer assisted education of the subject of permutation combination of mathematics on 8th grade students? level of achievement, and attitudes]. Yeditepe University: Yüksek Lisans Tezi [Master's thesis].

Arslan, A. (2008). Web destekli öğretimin ve ögrretimsel materyal kullanımının ögrencilerin matematik kaygisina, tutumuna ve başarisina etkisi [The effects of web supported instruction and use of instructional materials on students? mathematics anxieties, attitudes, and achievements]. Marmara University: Doktora Tezi [Doctoral thesis].

Asuman, Y. (2007). Illköğretim 2. sinuf seviyesinde bilgisayar destekli ĕgitici matematik oyunlarının başarlya ve kalıcılı̆̆a etkisi [The effect of computer assisted educational mathematic games on the academic achivement for maths course and retention of primary school 2nd grade students']. Çukurova University: Yüksek Lisans Tezi [Master's thesis].

Aşıcı, F. (2014). İlköğretim 6. sınıf matematik dersi kesirler konusunun excel yardımıyla öğretiminin öğrencilerin akademik başarılarına ve tutumlarına etkisi [The effect of the teaching fractions with excel on mathematics achievements and attitudes of sixth-grade students]. Gazi University: Yüksek Lisans Tezi [Master's thesis].

Aydoğan, A. (2007). The effect of dynamic geometry use together with open-ended explorations in sixth grade students' Performances in polygons and similarity and congruency of polygons. Middle East Technical University: Yüksek Lisans Tezi [Master's thesis].

Baki, A., \& Özpınar, İ. (2007). Logo destekli geometri öğretimi materyalinin öğrencilerin akademik başarılarına etkileri ve öğrencilerin uygulama ile ilgili görüşleri [The effects of logo supported geometry teaching material on the academic success of students and the students' opinions on the application]. Çukurova University Faculty of Education Journal, 3(34), 153-163.

Balkan, İ. (2013). Bilgisayar destekli öğretimin, ilköğretim 7. Sinıf ögrencilerinin matematik dersi "tablo ve grafikler" alt ögrenme alanındaki, akademik başarılarına ve tutumlarina etkisi [The effect of computer assisted instruction on academic achievements and attitudes of seventh-grade students in the field of mathematics 'Charts and Graphics']. Gazi University: Yüksek Lisans Tezi [Master's thesis].

Barutçu Akyar, K. (2010). Öklid geometrisi öğretiminde dinamik geometri yazılımları kullanımının 11. sınıf ögrencilerinin geometriye yönelik tutumlarına ve akademik başarllarına etkileri [The effect of utilizing the dynamic geometry software in geometry 
teaching on 11th grade students, their academic achievement, and their attitude towards geometry]. Dokuz Eylul Üniversity: Yüksek Lisans Tezi [Master's thesis].

Başaran Şimşek, E. (2012). Dinamik geometri yazılımı kullanmanın ilköğretim 6. Sınıf ögrencilerinin matematik dersindeki akademik başarllarına ve uzamsal yeteneklerine etkisi [The effect of utilizing the dynamic geometry software on 6th grade elemantary school students, their academic standings on mathematics lesson and their spatial ability]. Gazi University: Yüksek Lisans Tezi [Master's thesis].

Bayturan, S. (2011). Ortä̈ğretim matematik ĕgitiminde bilgisayar destekli ögretimin, ögrencilerin başarıları, tutumları ve bilgisayar öz-yeterlik algıları üzerindeki etkisi [The effect of computer- assisted instruction on the achievement, attitude, and computer self-efficacy of students in mathematics education]. Dokuz Eylul University: Doktora Tezi [Doctoral thesis].

Bayturan, S., Keşan, C. (2012). The effect of computer-assisted instruction on the achievement and attiudes towards mathematics of students in mathematics education. International Journal of Global Education, 2(1), 50-57.

Birgin, O., Kutluca, T., \& Gürbüz, R. (May, 2007). Yedinci sını matematik dersinde bilgisayar destekli ögretimin ögrenci başartsina etkisi [The effects of computer assisted instruction on the students' achievement in mathematics at seventh grade]. 8th International Educational Technology Conference, Eskişehir

Boyraz, Ş. (2008). The effects of computer based instruction on seventh grade students' spatial abllity, attıtudes toward geometry, mathematıcs and technology. Middle East Technical University: Yüksek Lisans Tezi [Master's thesis].

Budak, S. (2010). Çokgenler konusunun bilgisayar destekli öğretiminin 6. sinıf ögrrencilerinin akademik başarllarına ve bilgisayar destekli geometri ögretimine yönelik tutumlarına etkisi [The effects of computer aided education about polygons on 6. grade students? academic success]. Eskişehir Osman Gazi University: Yüksek Lisans Tezi [Master's thesis].

Bulut, M. (2009). İşbirliğine dayalı yapılandırmacı öğrenme ortamlarında kullanılan bilgisayar cebiri sistemlerinin matematiksel düşünme, öğrenci başarısina ve tutumuna etkisi [The effect of computer algebra systems in the constructivist approach based cooperative learning environment on mathematical thinking and academic performance of students]. Gazi University: Doktora Tezi [Doctoral thesis].

Buran, E. (2005). İkinci dereceden denklemler ve fonksiyonların gerçekci problem durumları ile ögrretilmesinde teknoloji destekli ve geleneksel yöntemlerin etkililiği [The Effect of technology based and traditional methods on teaching of quadratic equations and functions through pralistic problem situations]. Abant İzzet Baysal University: Yüksek Lisans Tezi [Master's thesis].

Çelik, H., Çevik, M. (September 2011). Illköğretim 7. Sınıföğrencilerinin "istatistik ve olasılık” ünitesini ögrrenmeleri üzerinde bilgisayar destekli ögretimin etkisi [The effect of computer-assisted instruction on teaching the unit of "probability and statistics" to 7th grade primary school students]. 5. International Computer \& Instructional Technologies Symposium. Elazı̆g.

Çubuk, Ş. (2004). Matematik ögretiminde "permütasyon ve olasılık" konusunun bilgisayar destekli ögrretim materyalleri ile ögretilmesinin ögrrenci başarisina etkisi [The effect of teaching the subject of "permutation and probability" in mathematics teaching with computer aided teaching materials on student success]. Marmara University: Yüksek Lisans Tezi [Master's thesis].

Demir, V. (2010). Cabri 3d dinamik geometri yazılımının, geometrik düşünme ve akademik başarı üzerine etkisi [ The effect of cabri 3d dynamic geometry software on geometric 
thinking and academic achievement]. Marmara University: Yüksek Lisans Tezi [Master's thesis].

Doğan, N. (2009). Bilgisayar destekli istatistik öğretiminin başarıya ve istatistiğe karşı tutuma etkisi [The effect of computer-assisted statistics instruction on achievement and attitudes toward statistics]. Education and Science, 34(154), 1-15.

Efendioğlu, A. (2006). Anlamlı ögrenme kuramına dayalı olarak hazırlanan bilgisayar destekli geometri programının ilköğretim dördüncü sınıf öğrencilerinin akademik başarılarına ve kalıcilı̆̆a etkisi [The effects of computer-based geometry programme based on meaningful learning on the academic achievement and retention of learning of primary school fourth grade students]. Çukurova University: Yüksek Lisans Tezi [Master's thesis].

Egelioğlu, H. C. (2008). Dönüşüm geometrisi ve dörtgensel bölgelerin alanlarının bilgisayar destekli ögretilmesinin başarlya ve epistemolojik inanca etkisi [Computer based education has an influence on success and epistemological belief in teaching of sub learning zones of transformation geometry and areas of quadrangle zones]. Marmara University: Yüksek Lisans Tezi [Master's thesis].

Ekici, F. (2008). Akıllı tahta kullanımının ilköğretim öğrencilerinin matematik başarılarına etkisi [Effects of smart board usage on primary school maths students' success]. Marmara University: Yüksek Lisans Tezi [Master's thesis].

Erdoğan, B. (2014). Dijital sınıfin akademik başarıya, çevrimiçi teknolojileri öz yeterlik algisina ve motivasyona etkisi [The effect of digital classroom upon academic success, technology self-efficacy and motivation]. Gazi University: Yüksek Lisans Tezi [Master's thesis].

Erginbaş, Ş. (2009). Teknoloji destekli matematik öğretiminin sınıf yönetiminin öğrenci özellikleri açısından etkililiği [The effects of technology-supported mathematics instruction on student characteristics of classroom management]. Süleyman Demirel Üniversity: Yüksek Lisans Tezi [Master's thesis].

Eryiğit, P. (2010). Üç boyutlu dinamik geometri yazılımı kullanımının 12. Sinıf ögrencilerinin akademik başarllarl ve geometri dersine yönelik tutumlarına etkileri [The effect of utilizing the three-dimensional dyinamic geometry software in geometry teaching on 12th grade students, their academic standings, their attitude towards geometry]. Dokuz Eylül University: Yüksek Lisans Tezi [Master's thesis].

Esen, B. (2009). Matematik ĕgitiminde ilköğretim 6. sinıflarda olasılık konusunun öğretiminde bilgisayar destekli eğitimin rolü [The role of computer assisted instruction in the teaching of probability in sixth grade students in mathematics education]. Selçuk University: Yüksek Lisans Tezi [Master's thesis].

Fırat, S. (2011). Bilgisayar destekli eğitsel oyunlarla gerçekleştirilen matematik öğretiminin kavramsal etkisi [The effect of mathematics teaching performed through educational computer games on conceptual learning]. Adıyaman University: Yüksek Lisans Tezi [Master's thesis].

Gelibolu, M. (2008). Gerçekçi matematik ĕ̌itimi yaklaşımıyla geliştirilen bilgisayar destekli mantı ögretimi materyallerinin 9. sinıf matematik dersinde uygulanmasının değerlendirilmesi [Evaluation of application of computer assisted logic instruction materials developped in realistic mathematics approach in 9th grade mathematics lessons]. Ege University: Yüksek Lisans Tezi [Master's thesis].

Genç, G. (2010). Dinamik geometri yazılımı ile 5. sınıf çokgenler ve dörtgenler konularının kavratılmasi [Teaching 5th grade polygon and quadrangle subjects through dynamic geometry software] Adnan Menderes University: Yüksek Lisans Tezi [Master's thesis].

Gökçül, M. (2007). Keller'ın arcs güdülenme modeline dayalı bilgisayar yazılımının matematik ögretiminde başarl ve kalıcıliğa etkisi [The Effects of computer software based on 
keller's arcs motivational model on the academic achievement and retention at teaching of mathematics]. Çukurova University: Yüksek Lisans Tezi [Master's thesis].

Gülbağc1, H. (2009). İlkögretim 7. Sinıf dörtgenler konusunun ögretiminde dinamik geometri yazllimlarinin etkisi [The effect of dynamic geometry software on seventh grade students" understanding of quadrilaterals]. Ankara University: Yüksek Lisans Tezi [Master's thesis].

Gündüz, Ş., Emlek, B., \& Bozkurt, A. (May, 2008). Computer aided teaching trigonometry using dynamic modelling in high school. 8th International Educational Technology Conference, Eskişehir.

Gürsul, F. (2008). Çevrimiçi ve yüzyüze problem tabanlı öğrenme yaklaşımlarının ögrrencilerin başarllarına ve matematiğe yönelik tutumlarına etkisi. [The effects of online and face to face problem-based learning approaches on student's academic achievement, their attitudes towards mathematics]. Ankara University: Doktora Tezi [Doctoral thesis].

Helvac1, B. T. (2010). Bilgisayar destekli ögretimin, ilköğretim 6. sinıf ögrencilerinin matematik dersi "çokgenler" konusundaki akademik başarılarına ve tutumlarına etkisi [Computer-aided instruction, elementary 6th grade students' mathematics achievement and attitudes in polygons effects topics]. Gazi University: Yüksek Lisans Tezi [Master's thesis].

Kabaca, T. (2006). Limit kavramının ögretiminde bilgisayar cebiri sistemlerinin etkisi. [The effect computer algebra systems on teaching limit concept]. Gazi Üniversity: Doktora Tezi [Doctoral thesis].

Kan, O. (2014). Geogebra destekli ögretimin lineer cebir dersine ait bazı konularda akademik başarl üzerine etkisi [The effect of GeoGebra assisted instruction on academic achievement in some issues of linear algebra course]. Necmettin Erbakan University: Yüksek Lisans Tezi [Master's thesis].

Karakuş, Ö. (2008). Bilgisayar destekli dönüşüm geometrisi öğretiminin öğrenci erişisine etkisi [The effects on the students' success of computer-based transformation geometry learning]. Eskişehir Osman Gazi University: Yüksek Lisans Tezi [Master's thesis].

Karasel, N., Ayda, O., \& Tezer, M. (2009, May). Effect of problem posing method in mathematics education with computer assisted pictures on student success and attitudes. 9th International Educational Technology Conference. Ankara.

Kaya, G. (2013). Matematik derslerinde akıllı tahta kullanımının öğrencilerin dönüşüm geometrisi üzerindeki başarlarına etkisi [The effects of using interactive whiteboards on students' achievement of transformational geometry in mathematics lessons]. Gazi University: Yüksek Lisans Tezi [Master's thesis].

Kaya, D., Keşan, C., \& İzgiol, D. (2013). The effect of internet-based education on student success in teaching of 8 th grade triangles subject. Turkish Online Journal of Distance Education, 14(1), 202-210.

Kepçeoğlu, İ. (2010). Geogebra yazılımıyla limit ve süreklilik öğretiminin ögretmen adaylarının başarısına ve kavramsal ögrenmelerine etkisi [The effect of using geogebra in teaching limit and continuity on pre-service teachers' academic achievement and conceptual learning]. Marmara University: Yüksek Lisans Tezi [Master's thesis].

Kesicioğlu, O. (2011). Doğrudan öğretim yöntemiyle hazırlanan eğitim programının ve bu yönteme göre hazırlanan bilgisayar destekli eğitim programının okul öncesi çocuklarının geometrik şekil kavramlarını ögrenmelerine etkisinin incelenmesi [An analysis of the impact of an instructional program designed with direct instruction method and of a computer assisted instructional program designed in accordance with this method on preschoolers' geometric figures concepts learning]. Gazi Üniversity: Doktora Tezi [Doctoral thesis]. 
Kılıç, R. (2007). Webquest destekli işbirlikçi öğrenme yönteminin matematik dersindeki tutum ve erişiye etkisi [The Effects of webquest assisted cooperative learning method on the attitudes and achievement towards mathematics lesson]. Eskişehir Osmangazi University: Yüksek Lisans Tezi [Master's thesis].

Kurak, Y. (2009). Dinamik geometri yazılımı kullanımının öğrencilerin dönüşüm geometri anlama düzeylerine ve akademik başarllarına etkisi [The effects of using dynamic geometry software on students? understanding levels of transformation geometry and their academic successes]. Karadeniz Teknik University: Yüksek Lisans Tezi [Master's thesis].

Kutluca, T. (2009). İkinci dereceden fonksiyonlar konusu için tasarlanan bilgisayar destekli ögrenme ortamının değerlendirilmesi [Evaluation of a computer assisted learning environment designed for the subject of quadratic functions]. Karadeniz Teknik University: Doktora Tezi [Doctoral thesis].

Küslü, F. (2015). Bilgisayar destekli matematik öğretiminin 8. sinıföğrencilerinin "prizmalar" konusundaki başarısina etkisi [The effect of computer assisted mathematics teaching on the success of the 8th grade students in the topic of 'prisms']. Sakarya University: Yüksek Lisans Tezi [Master's thesis].

İçel, R. (2011). Bilgisayar destekli öğretimin matematik başarısına etkisi: Geogebra örneği [Effects of computer-based teaching on students' mathematics achievements: Example of GeoGebr]. Selçuk Üniversity: Yüksek Lisans Tezi [Master's thesis].

İnam, A. (2014). Ortaokul 5. Sinıf matematg்k uygulamaları dersinin web destekli öğretiminin ögrenci performans ve motivasyonuna etkisi ile öğrenci görüşlerinin değerlendirilmesi [The effect of teaching web-based applications of maths subject for 5th grade students' performance and motivation in accordance with the evaluation of students' opinions]. Gazi University: Yüksek Lisans Tezi [Master's thesis].

İzgiol, D. (2014). Teknoloji destekli çoklu temsil temelli öğretimin ögrencilerin lineer cebir ögrenimine ve matematiğe yönelik tutumlarına etkisi [A study on the effects of technology-assisted multiple representations-based instruction on students' linear algebra learning and attitudes towards mathematics]. Dokuz Eylül Üniversity: Yüksek Lisans Tezi [Master's thesis].

Akar, Ü., \& Hacısalihoğlu Karadeniz, M. (2014). Dinamik geometri yazılımının açıortay ve kenarortay öğretiminde meslek lisesi öğrencilerinin başarılarına etkisi [The effect of dynamic geometry software on the vocational high school students' succes for teaching bisector and the median concepts]. Journal of Computer and Education Research, 2(4), 74-90.

Halat, E. (2008). The effects of designing Webquests on the motivation of pre-service elementary school teachers. International Journal of Mathematical Education in Science and Technology, 39(6), 793-802.

Memişoğlu, B. (2005). Matematik öğretiminde bilişim teknolojilerinin kullanımı [Using of technologicaly informatics at the education of mathematics]. Balıkesir University: Yüksek Lisans Tezi [Master's thesis].

Mercan, M. (2012). Ilköğretim 7. Sinıf matematik dersine ait "dönüşüm geometrisi” alt ögrenme alanının ögretiminde, dinamik geometri yazılımı geogebra'nın kullanımının ögrenci başarlsina etkisi [The effects of use of a dynamic geometry software geogebra in teaching the subject of transformation geometry in seventh grade math class in a primary school on students' achievement and retention levels]. Gazi University: Yüksek Lisans Tezi [Master's thesis].

Oğuz, A. (2008). Denklemler alt ögrenme alanında cd destekli öğretimin öğrenci başarısına etkisi [The effects of student success of materials prepared according to CD-supported 
on equations learning subsection]. Marmara University: Yüksek Lisans Tezi [Master's thesis].

Önal, N., \& Göloğlu Demir, C. (2012). Yedinci sınıflarda bilgisayar destekli geometri öğretiminin öğrenci başarısına etkisi [The effect of computer assisted geometry instruction on seventh grade school students' achievement]. Turkish Journal of Education, 2(1), 19-28.

Öner G., Yıldırım, İ., \& Bars, M. (2014). Harmanlanmış öğrenme yaklaşımının matematik dersi 2. Dereceden denklemler alt öğrenme alanında öğrenci başarısına etkisi [The effect of blended learning on students' achievement for the topic of quadratic equation in mathematics education]. Journal of Computer and Educational Research, 2(4), 152165.

Önür, Y, (2008). Effects of graphing calculators on eighth grade students' achievement in graphs of linear equations and concept of slope. Middle East Technical University: Doktora Tezi [Doctoral thesis].

Öz, A. (2012). Somut materyallergn ve geometer's sketchpad yazllımının derslerde kullanımının ögretmen adaylarının geometri başarılarına etkisinin incelenmesi [Investigating the effect of using concrete materials and the Geometer's Sketchpad software on pre-service teachers' geometry achievement]. Gaziantep University: Yüksek Lisans Tezi [Master's thesis].

Özçakır, B. (2013). The effects of mathematıcs instructıon supported by dynamıc geometry activities on seventh grade students' achievement in area of quadrilaterals. Middle East Technical University: Yüksek Lisans Tezi [Master's thesis].

Özdoğan, E. (2008). İşbirlikli öğrenme yönteminin ilköğretim 4. Sinıfmatematik ögrretiminde ögrenci tutum ve başarısına etkisi: bilgisayar destekli işbirlikli ögrenme ve küme destekli bireyselleştirme tekniği [The effect of cooperative learning method to the fourth-grade students' attitude and achievement on mathematic learning: the computer supported collobarotive learning (CSCL) and team assisted individualization method (TAI)]. Ege University: Yüksek Lisans Tezi [Master's thesis].

Özdemir, A., \& Tabuk, M. (2003). Matematik dersinde bilgisayar destekli öğretimin öğrenci başarıları ve tutumlarına etkisi [The Effect of computer aided instruction to the students' achievement and attitude in mathematics]. Bolu Abant Izzet Baysal University Journal of Faculty of Education, 4(1), 41-52.

Özen, D. (2010). İlköğretim 7. Sinıf geometri ögretiminde dinamik geometri yazılımlarının ögrencilerin erişi düzeylerine etkisi ve ögrrenci görüşlerinin değerlendirilmesi [The effect of dynamic geometry softwares on students? academic achievement level and the assessment of the students? opinions in geometry instruction at 7th grade]. Dokuz Eylül University: Yüksek Lisans Tezi [Master's thesis].

Özkök, E. (2010). Gagne’nin ögrretim modeliyle hazırlanan öğretim yazılımının ilköğretim 8. sınıf ögrencilerinin matematik dersi kareköklü sayılar konusundaki akademik başarısına ve ögrenci tutumlarına etkisi [The effect of the teaching software which is prepared with Gagne's Teaching Model on the students' academic success rate in mathematics subject on square rooted numbers topic and students' attitudes at the eighth grade in primary school]. Gazi University: Yüksek Lisans Tezi [Master's thesis].

Öztürk, T. (2011). Matematik öğretiminde bilgisayar destekli ögretim yöntemiyle hazırlanan animasyon tekniğinin kullanımı [The usage of animation technique prepared with computer assisted method in teaching mathematics]. Firat University: Yüksek Lisans Tezi [Master's thesis].

Öztürk, B. (2012). Geogebra matematik yazllımının ilköğretim 8. sınıf matematik dersi trigonometri ve ĕgim konuları öğretiminde, öğrenci başarısına ve Van Hiele geometri düzeyine etkisi [Effect of mathematics software GeoGebra mathematics lesson on 
achievement and Van Hiele levels of geometric of 8th grades in teaching of trigonometry and slope issues]. Sakarya University: Yüksek Lisans Tezi [Master's thesis].

Özyurt, Ö. (2009). Uyarlanabilir zeki web tabanlı matematik öğrenme ortamının tasarlanması, uygulanması ve değerlendirilmesi [The design, implementation and evaluation of an adaptive intelligent web-based mathematics learning environment]. Karadeniz Technical University: Doktora Tezi [Doctoral thesis].

Şataf, H. (2010). Bilgisayar destekli matematik öğretiminin ilköğretim 8. sinıf ögrrencilerinin "dönüşüm geometrisi" ve "üçgenler" alt ögrenme alanındaki başarısı ve tutuma etkisi (Isparta örneği) [Effect of computer-assisted mathematics teaching on achievement and attitude of 8th graders in transformation geometry and triangles sub-learning areas sample isparta primary school]. Sakarya Üniversity: Yüksek Lisans Tezi [Master's thesis].

Selçik, N., \& Bilgici, G. (2011). Geogebra yazılımının öğrenci başarısına etkisi [The effect of the geogebra software on students' academic achievement]. Kastamonu Education Journal, 19(3), 913-924.

Şen, N. (2010). Illköğretim altıncı sınıf matematik dersinde bilgisayar destekli sezgisel dü̧̈ünme kontrollü olasılık öğretiminin öğrencilerin akademik başart ve sezgisel düşünme düzeylerine etkisi [The effect of computer-based probabilty unit designed for the controlling of intuitive thinking on academic success, intuitive thinking, and retention]. Çukurova Üniversity: Yüksek Lisans Tezi [Master's thesis].

Şimşek, Ö. (2010). Web destekli matematik öğretiminde kullanılan video derslerin ögrenenlerin türev başarllarına etkisi ve ögrenenlerin video derslere ilişkin görüşleri [The effect of video lectures used in web enhanced mathematics instruction on students' derivative achievement and views of students about the video lectures]. Ege University: Yüksek Lisans Tezi [Master's thesis].

Takunyacı, M. (2007). İlköğretim 8. Sınıf öğrencilerinin geometri başarısında bilgisayar destekli ögretimin etkisi [The effect of computer assisted instruction on students' achievement on geometry of 8th grade]. Sakarya University: Yüksek Lisans Tezi [Master's thesis].

Tataroğlu, B. (2009). Matematik ögretiminde akıllı tahta kullanımının 10. Sinıf öğrencilerinin akademik başarıları, matematik dersine karşı tutumları ve öz-yeterlik düzeylerine etkileri [The effect of utilizing the smart board in mathematics teaching on 10th grade students, their academic standings, their attitude towards mathematics and their selfefficacy levels]. Dokuz Eylül University: Yüksek Lisans Tezi [Master's thesis].

Tayan, E. (2011). Doğrusal denklemler ve grafikleri konusunun öğretiminde bilgisayar destekli ögretim yönteminin başarıya etkisi [The effect of computer assisted instruction method on student success in teaching of linear equations and graphics]. Atatürk University: Yüksek Lisans Tezi [Master's thesis].

Toker, Z. (2008). The effect of using Dynamic Geometry Software while teaching by guided discovery on students' geometric thinking levels and achievement. Middle East Technical University: Yüksek Lisans Tezi [Master's thesis].

Topaloğlu, İ. (2011). Cabri 3d ile yapılan ders tasarımlarının öğrencilerin uzamsal görselleme ve başarılarına etkisinin incelenmesi [The search of the effect on student's visual and extent success with the lesson conception by using Cabri 3d]. Marmara University: Yüksek Lisans Tezi [Master's thesis].

Tuluk, G. (2007). Fonksiyon kavramının öğretimine bilgisayar cebiri sistemlerinin etkisi [The effect of computer algebra systems in the teaching of function concept]. Gazi University: Doktora Tezi [Doctoral thesis].

Tural Sönmez, M., \& Dinç Artut, P. (June, 2012). Web üzerinden sunulan eğitsel matematik oyunlarının kesirler ve ondalık sayılara ilişkin öğrenci başarısına etkisi [The effect of 
educational math games on the web on student achievement regarding fractions and decimals]. X. National Science and Mathematics Education Congress, Niğde

Turgut, M. (2010). Teknoloji destekli lineer cebir öğretiminin ilköğretim matematik ögretmen adaylarinin uzamsal yeteneklerine etkisi [The effect of technology assisted linear algebra instruction on pre-service primary mathematics teachers' spatial ability]. Dokuz Eylul University: Doktora Tezi [Doctoral thesis].

Tural Sönmez, M. (2012). 6. Sinıf matematik derslerinde web üzerinden sunulan eğitsel matematik oyunlarının ögrenci başarısına etkisi [The effects of instruction with webbased games on sixth grade students' academic achievement in fractions and decimals subject in mathematics lesson]. Çukurova University: Yüksek Lisans Tezi [Master's thesis].

Tutak, T. (2008). Somut nesneler ve dinamik geometri yazılımı kullanımının ögrencilerin bilişsel ögrenmelerine, tutumlarına ve Van Hiele geometri anlama düzeylerine etkisi [The effects of using concrete materials and dynamic geometry software on students' cognitive learning, attitudes, and understanding levels of Van Hiele geometry]. Karadeniz Technical University: Doktora Tezi [Doctoral thesis].

Uygan, C. (2011). Katı cisimlerin ögretiminde google sketchup ve somut model destekli uygulamaların ilköğretim matematik öğretmeni adaylarının uzamsal yeteneklerine etkisi [The effects of google sketchup and concrete model based applications in solids education on spatial ability of pre-service elementary mathematics teachers]. Eskişehir Osmangazi University: Yüksek Lisans Tezi [Master's thesis].

Uzun, N. (2013). Dinamik geometri yazılımlarının bilgisayar destekli ögretim ve akıllı tahta ile zenginleştirilmiş ögrenme ortamlarında kullanımının öğrencilerin akademik başarisina, uzamsal görselleştirme becerisine ve uzamsal düşünme becerisine ilişkin tutumlarina etkisi [Effect of using dynamic geometry software in computer based and enhanced with smart board learning environments on student's academic achievement, spatial visualization ability and spatial thinking atitude]. Gazi University: Yüksek Lisans Tezi [Master's thesis].

Uygun, M. (2008). Bilgisayar destekli bir öğretim yazılımının ilköğretim 4. Sinı öğrencilerinin kesirler konusundaki başarı ve matematiğe karşı tutumuna etkisinin incelenmesi [Investigation of the effects of an educational software on 4th grade primary school students? attitudes towards mathematics and their achievements on fractions]. Abant İzzet Baysal University: Yüksek Lisans Tezi [Master's thesis].

Ünlü, M. (2007). Problem çözme ve buluş yoluyla öğretim kuramına göre geliştirilmiş web tabanl eğitimin ögrenci başarlsina etkisi [The effects of web-based training environment based upon problem solving and discovery learning theory on the success of the students]. Gazi University: Yüksek Lisans Tezi [Master's thesis].

Yahşi Sarı, H. (2012). İlköğretim 7. Sinıf matematik dersi dönüşüm geometrisi” alt öğrenme alanının ögretiminde dinamik geometri yazllımlarından sketchpad ile geogebra'nın kullanımlarının ögrencilerin başarısına ve ögrrenmelerin kalıcilı̆̆ına etkilerinin karşılaştırılması [Primary 7th grade mathematics courses teaching the learning area of the lower of dynamic geometry using the software of the rotation geometry with geometer's sketchpad and Geogebra to compare the effects on students' success and permanency of learning]. Gazi University: Yüksek Lisans Tezi [Master's thesis].

Yazlık, D. (2011). İlköğretim 7. Sinıflarda cabri geometri plus ıl ile dönüşüm geometrisi ögretimi [Teaching transformation geometry with Cabri Geometry Plus II in seventh grade]. Selçuk University: Yüksek Lisans Tezi [Master's thesis]. 
Yıldız, Z. (2009). Geometrik cisimlerin yüzey alanları ve hacimleri konularında bilgisayar destekli ögretimin ilkögretim 8. Sınıf ögrenci tutumu ve başarısına etkisi [The effect of computer assisted instruction related with the surface area and volume of geometrical objects onto the achievement and the attitude of eight grade primary school students]. Gazi University: Yüksek Lisans Tezi [Master's thesis].

Yorgancı, S., \& Terzioğlu, Ö. (2013). Matematik öğretiminde akıllı tahta kullanımının başarıya ve matematiğe karşı tutuma etkisi [The effect of using interactive whiteboard in mathematics instruction on achievement and attitudes toward mathematics]. Kastamonu Education Journal, 21(3), 919 - 930.

Yorganc1, S. (2014). Web tabanlı uzaktan eğitim yönteminin öğrencilerin matematik başarılarına etkileri [The effects of web-based distance education method on students' mathematics achievements]. Kastamonu Education Journal, 23(3), 14011420 .

Uzun, P. (2014). Geogebra ile öğretimin 7. Sinıf öğrencilerinin akademik başarlarına ve geometriye yönelik tutumlarına etkisi [The effect of instruction with geogebra on 7th grade students' academic achievement and attitudes toward geometry]. Kastamonu University: Yüksek Lisans Tezi [Master's thesis].

Zengin, Y. (2011). Dinamik matematik yazılımı Geogebra'nın öğrencilerin başarılarına ve tutumlarına etkisi [The effect of dynamic mathematics software GeoGebra on students' achievement and attitude]. Kahramanmaraş Sütçü İmam Üniversity: Yüksek Lisans Tezi [Master's thesis]. 